\title{
Orbital stability of periodic waves for the nonlinear Schrödinger equation
}

\author{
Thierry Gallay \\ Institut Fourier \\ Université de Grenoble I \\ B.P. 74 \\ 38402 Saint-Martin-d'Hères, France \\ Mariana Hărăguş \\ Département de Mathématiques \\ Université de Franche-Comté \\ 16 route de Gray \\ 25030 Besançon, France
}

\begin{abstract}
The nonlinear Schrödinger equation has several families of quasi-periodic travelling waves, each of which can be parametrized up to symmetries by two real numbers: the period of the modulus of the wave profile, and the variation of its phase over a period (Floquet exponent). In the defocusing case, we show that these travelling waves are orbitally stable within the class of solutions having the same period and the same Floquet exponent. This generalizes a previous work [12] where only small amplitude solutions were considered. A similar result is obtained in the focusing case, under a non-degeneracy condition which can be checked numerically. The proof relies on the general approach to orbital stability as developed by Grillakis, Shatah, and Strauss 15 16, and requires a detailed analysis of the Hamiltonian system satisfied by the wave profile.
\end{abstract}

Running head: Periodic waves in the NLS equation

Corresponding author: Thierry Gallay, Thierry.Gallay@ujf-grenoble.fr

Keywords: Nonlinear Schrödinger equation, periodic waves, orbital stability 


\section{Introduction}

This paper is devoted to the stability analysis of the quasi-periodic travelling wave solutions of the cubic nonlinear Schrödinger (NLS) equation

$$
\mathrm{i} U_{t}(x, t)+U_{x x}(x, t)+\gamma|U(x, t)|^{2} U(x, t)=0, \quad x \in \mathbb{R}, \quad t \in \mathbb{R},
$$

where $\gamma \in\{-1 ; 1\}$ and $U(x, t) \in \mathbb{C}$. Eq.(1.1) is a universal envelope equation describing the propagation of weakly nonlinear waves in dispersive media (see [20] for a comprehensive introduction). The nonlinearity in (1.1) is "attractive" if $\gamma=+1$ (focusing case) and "repulsive" if $\gamma=-1$ (defocusing case). In both cases Eq.(1.1) has a family of quasi-periodic travelling waves of the form

$$
U(x, t)=\mathrm{e}^{\mathrm{i}(p x-\omega t)} V(x-c t), \quad x \in \mathbb{R}, \quad t \in \mathbb{R},
$$

where $p, \omega, c$ are real parameters and $V: \mathbb{R} \rightarrow \mathbb{C}$ is a periodic function. The simplest elements of this family are the plane waves, for which $V$ is identically constant and $p, \omega$ satisfy the dispersion relation $p^{2}=\omega+\gamma|V|^{2}$. It is well-known and easy to verify that the plane waves are dynamically stable in the defocusing case, and unstable (if $V \neq 0$ ) in the focusing case 22, 12. We shall therefore concentrate on the less explored situation where $V$ is a nontrivial periodic function. In that case, we shall refer to (1.2) as a periodic wave, although $U(x, t)$ is in general a quasi-periodic function of both $x$ and $t$.

The number of parameters in (1.2) can be reduced if we use the symmetries of Eq.(1.1). We recall that the NLS equation is invariant under the following transformations:

(i) $U(x, t) \mapsto U(x, t) \mathrm{e}^{\mathrm{i} \varphi}, \varphi \in \mathbb{R}$ (Phase invariance);

(ii) $U(x, t) \mapsto U(x+\xi, t), \xi \in \mathbb{R}$ (Translation invariance);

(iii) $U(x, t) \mapsto \mathrm{e}^{-\mathrm{i}\left(\frac{v}{2} x+\frac{v^{2}}{4} t\right)} U(x+v t, t), v \in \mathbb{R}$ (Galilean invariance);

(iv) $U(x, t) \mapsto \lambda U\left(\lambda x, \lambda^{2} t\right), \lambda>0$ (Dilation invariance).

If $U(x, t)$ is a periodic wave as in (1.2), we can use the Galilean invariance to transform it into a solution of the same form with $c=0$. Then, using the dilation invariance, we can further assume that $\omega \in\{-1 ; 0 ; 1\}$. It follows that $U(x, t)=\mathrm{e}^{-\mathrm{i} \omega t} W(x)$, where $W(x)=\mathrm{e}^{\mathrm{i} p x} V(x)$ is a solution of the ordinary differential equation

$$
W_{x x}(x)+\omega W(x)+\gamma|W(x)|^{2} W(x)=0, \quad x \in \mathbb{R} .
$$

The bounded solutions of (1.3) are completely classified for all values of the parameters $\omega, \gamma$. The simplest ones are the plane waves $W(x)=A \mathrm{e}^{\mathrm{i} p x}$ where $p \in \mathbb{R}, A \in \mathbb{C}$ and $p^{2}=\omega+\gamma|A|^{2}$. The periodic waves correspond to quasi-periodic solutions of (1.3) of the form $W(x)=r(x) \mathrm{e}^{\mathrm{i} \varphi(x)}$, where $r, \varphi$ are real functions with the property that $r$ and $\varphi_{x}$ are periodic with the same period. It turns out that Eq.(1.3) has a four-parameter family of such solutions, both in the focusing and in the defocusing case (see Sections 2 and 4 below). Actually, if $\gamma=-1$, we must assume that $\omega=1$ otherwise (1.3) has no nontrivial bounded solutions; if $\gamma=+1$, Eq.(1.3) has quasi-periodic 
solutions for all values of $\omega$, but we shall only consider the generic cases $\omega= \pm 1$. If $\gamma \omega<0$, in addition to plane waves and periodic waves, there exist pulse-like solutions of (1.3) which are homoclinic as $x \rightarrow \pm \infty$ to a plane wave or to the zero solution. The most famous one (if $\gamma=1$ and $\omega=-1)$ is the ground state $W(x)=\sqrt{2} / \cosh (x)$ which corresponds to the solitary wave of the focusing NLS equation.

In contrast to the plane waves or the solitary waves which have been extensively studied [7, 21, 22], relatively little seems to be known about the stability of periodic waves. Spectral stability with respect to long-wave disturbances has been examined by Rowlands [19], who showed that periodic waves with real-valued profile are unstable in the focusing case and stable (at least in the longwave regime) in the defocusing case. In the latter case, spectral stability of the small amplitude periodic waves has been rigorously established in [12] using Bloch-wave analysis. Similar results were also obtained for certain NLS-type equations with spatially periodic potentials [6, 18. As for the nonlinear stability, the only result we are aware of is due to Angulo [1], who proved very recently that the family of dnoidal waves of the focusing NLS equation is orbitally stable with respect to perturbations which have the same period as the wave itself. We recall that the periodic waves of NLS with real-valued profile are called "cnoidal waves" when they have zero average over a period (like the Jacobian elliptic function $c n$ ), and "dnoidal waves" when they have nonzero average (like the elliptic function $d n$ ).

In this paper, we study the nonlinear stability of all periodic waves of (1.1), but we restrict ourselves to a specific class of perturbations which we now describe. Any quasi-periodic solution of (1.3) can be written in the form

$$
W(x)=\mathrm{e}^{\mathrm{i} p x} Q_{\text {per }}(2 k x), \quad x \in \mathbb{R},
$$

where $p \in \mathbb{R}, k>0$, and $Q_{\text {per }}: \mathbb{R} \rightarrow \mathbb{C}$ is $2 \pi$-periodic. Here $k=\pi / T$, where $T>0$ is the minimal period of $|W|$. The representation (1.4) is not unique, since we can add to $p$ any integer multiple of $2 k$ (and modify the periodic function $Q_{\text {per }}$ accordingly), but the Floquet multiplier $\mathrm{e}^{\mathrm{i} p T}$ is uniquely defined. Our purpose is to show that the periodic wave $U(x, t)=\mathrm{e}^{\mathrm{i}(p x-\omega t)} Q_{\text {per }}(2 k x)$ of (1.1) is stable within the class of solutions which have the same period $T=\pi / k$ and the same Floquet multiplier $\mathrm{e}^{\mathrm{i} p T}$. In other words, we restrict ourselves to solutions of (1.1) of the form $\mathrm{e}^{\mathrm{i}(p x-\omega t)} Q(2 k x, t)$, where $Q(\cdot, t)$ lies in the function space

$$
X=H_{\text {per }}^{1}([0,2 \pi], \mathbb{C})=\left\{Q \in H_{\text {loc }}^{1}(\mathbb{R}, \mathbb{C}) \mid Q(z)=Q(z+2 \pi) \text { for all } z \in \mathbb{R}\right\} .
$$

The advantage of this restricted setting is that nonlinear stability can be established by the standard variational method which has been developed originally to prove the orbital stability of solitary waves [3, 4, 21] (see also [1, 2]). However, the obvious drawback of this approach is that it does not give any information on the stability of the periodic waves with respect to non-periodic perturbations, a difficult question which remains essentially open.

With this perspective in mind, we shall put the emphasis on the defocusing case $\gamma=-1$, because we know from [19] that the periodic waves will be unstable in the focusing case if non-periodic perturbations are to be allowed. Our main result can be stated as follows: 
Theorem 1 (Orbital stability of periodic waves in the defocusing case)

Let $\gamma=-1, \omega=1$, and assume that $W(x)=\mathrm{e}^{\mathrm{i} p x} Q_{\mathrm{per}}(2 k x)$ is a solution of (1.3) with $p \in \mathbb{R}$, $k>0$, and $Q_{\text {per }} \in X$, as in (1.4). Then there exist $C_{0}>0$ and $\epsilon_{0}>0$ such that, for all $R \in X$ with $\|R\|_{X} \leq \epsilon_{0}$, the solution $U(x, t)=\mathrm{e}^{\mathrm{i}(p x-\omega t)} Q(2 k x, t)$ of the $N L S$ equation (1.1) with initial data $U(x, 0)=\mathrm{e}^{\mathrm{i} p x}\left(Q_{\text {per }}(2 k x)+R(2 k x)\right)$ satisfies, for all $t \in \mathbb{R}$,

$$
\inf _{\varphi, \xi \in[0,2 \pi]}\left\|Q(\cdot, t)-\mathrm{e}^{\mathrm{i} \varphi} Q_{\text {per }}(\cdot-\xi)\right\|_{X} \leq C_{0}\|R\|_{X} .
$$

This result is known to hold for small amplitude periodic waves [12, in which case the constants $C_{0}, \epsilon_{0}$ do not depend on the wave profile $Q_{\text {per }}$. Here we remove the smallness assumption, but our argument relies in part on the calculations made in [12] (see Lemma 3.3 below).

\section{Remarks}

1. Theorem 1 includes the situation where $|W|$ is constant, in which case $W$ is a plane wave rather than a periodic wave. Since stability is well-known for plane waves 22], we shall assume henceforth that $|W|$ is a nontrivial periodic function. In such case, we emphasize that the wavenumber $k$ which appears in (1.4) is always given by $k=\pi / T$, where $T>0$ is the minimal period of $|W|$. This is very important because our approach does not allow to prove the stability of periodic waves with respect to perturbations whose period is an integer multiple of $T$.

2. It is interesting to see what Theorem 1 means in the particular case of cnoidal waves. For such waves we have $W(x)=\mathrm{e}^{\mathrm{i} p x} Q_{\text {per }}(2 k x)$ where $p=k=\pi / T$ and $T>\pi$ is the minimal period of $|W|$. The Floquet multiplier $\mathrm{e}^{\mathrm{i} p T}$ is therefore equal to -1 , so that $W(x+T)=-W(x)$, for all $x \in \mathbb{R}$. In particular, $W$ is periodic with (minimal) period $L=2 T$. Theorem 1 then shows that the $L$-periodic cnoidal wave $U(x, t)=\mathrm{e}^{-\mathrm{i} t} W(x)$ is orbitally stable with respect to $L$-periodic perturbations $\widetilde{W}$ provided that $\widetilde{W}(x+L / 2)=-\widetilde{W}(x)$ for all $x \in \mathbb{R}$. As explained in [1, without this additional assumption the classical approach does not allow to prove the orbital stability of cnoidal waves with respect to perturbations which have the same period as the wave itself.

The rest of the paper is organized as follows. In Section 2 we recall the classification of the bounded solutions of (1.3) with $\gamma=-1$ and $\omega=1$. These solutions can be interpreted as the trajectories of an integrable Hamiltonian system with two degrees of freedhom, which is proved to be non-degenerate in the sense of KAM theory. We thereby answer a question raised by Bridges and Rowlands [5] in connexion with the stability of the quasi-periodic solutions of the Ginzburg-Landau equation.

Section 3 is devoted to the proof of Theorem [1. As indicated above, we follow the general approach of Grillakis, Shatah, and Strauss. The main difficulty is to verify the assumptions of the stability theorem in [16]. We first check that the second variation of the energy functional at the periodic wave has exactly one negative eigenvalue. This result has been established for small waves in 12, and a continuity argument allows to extend it to periodic waves of arbitrary size. We next consider the structure function (which is called " $d(\omega)$ " in [16]) and show, by a direct calculation, that its Hessian matrix has a negative determinant. Both properties together imply orbital stability.

Finally, in Section 4, we extend our results to the focusing NLS equation. The situation is more complicated here, because we have families of periodic waves for all values of $\omega \in\{-1 ; 0 ; 1\}$. By 
comparison with the spatially homogeneous rotating wave $U(x, t)=\mathrm{e}^{\mathrm{i} t}$, these periodic waves may be called "counter-rotating" if $\omega=+1$, "standing" if $\omega=0$, and "corotating" if $\omega=-1$. As was already mentioned, we restrict ourselves to the generic cases $\omega= \pm 1$. In both situations, we show that the Hamiltonian system corresponding to (1.3) is non-degenerate in the sense of KAM, and we deduce as in Section 3 that the second variation of the energy functional has exactly one negative eigenvalue. It follows that orbital stability holds provided the Hessian matrix of the structure function has a negative determinant (see Propositions 4.3 and 4.6 for precise statements). Unlike in the defocusing case, we do not give a complete proof of this property, because the calculations are excessively complicated. But the determinant is easy to evaluate numerically (or even analytically

in various parameter regimes), and it appears to be negative for all periodic waves. Assuming this to be true, we conclude that the analogue Theorem 1 holds in the focusing case too. Thus, in contrast to what happens when long-wave disturbances are considered [19, there is apparently no difference between the focusing and the defocusing case as far as periodic perturbations are considered.

Acknowledgements. The authors are indebted to A. De Bouard and L. Di Menza for fruitful discussions. This work was partially supported by the French Ministry of Research through grant ACI JC 1039.

\section{Properties of the periodic waves}

In this section, we study the bounded solutions of the stationary Ginzburg-Landau equation

$$
W_{x x}(x)+W(x)-|W(x)|^{2} W(x)=0,
$$

where $W: \mathbb{R} \rightarrow \mathbb{C}$. If we interprete the spatial variable $x \in \mathbb{R}$ as a "time", Eq. (2.1) becomes an integrable Hamiltonian dynamical system with two degrees of freedhom. The conserved quantities are the "angular momentum" $J$ and the "energy" $E$ :

$$
J=\operatorname{Im}\left(\bar{W} W_{x}\right), \quad E=\frac{1}{2}\left|W_{x}\right|^{2}+\frac{1}{2}|W|^{2}-\frac{1}{4}|W|^{4} .
$$

If $W$ is a solution of (2.1) with $J \neq 0$, then $W(x) \neq 0$ for all $x \in \mathbb{R}$, so that we can introduce the polar coordinates $W(x)=r(x) \mathrm{e}^{\mathrm{i} \varphi(x)}$. The invariants then become

$$
J=r^{2} \varphi_{x}, \quad E=\frac{r_{x}^{2}}{2}+\frac{J^{2}}{2 r^{2}}+\frac{r^{2}}{2}-\frac{r^{4}}{4} .
$$

Let $D \subset \mathbb{R}^{2}$ be the open region defined by

$$
D=\left\{(J, E) \in \mathbb{R}^{2} \mid J^{2}<4 / 27, E_{-}(J)<E<E_{+}(J)\right\},
$$

where the functions $E_{-}, E_{+}$are defined in (2.4) below (see also Fig. 1). Our starting point is the following well-known classification [5, 9, 11]: 


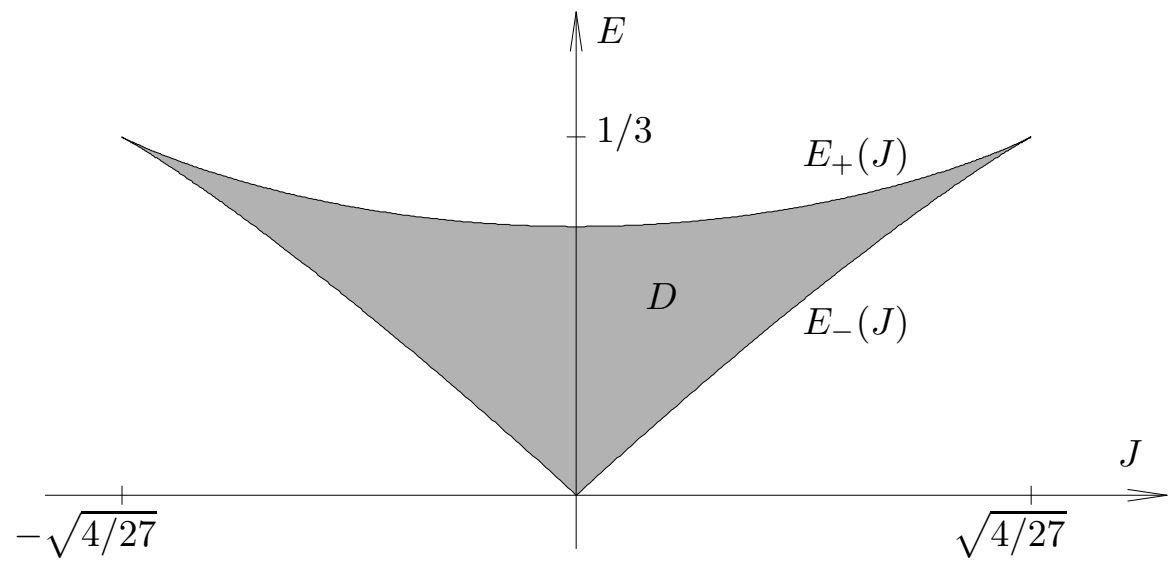

Fig. 1: The region $D$ in the parameter space for which Eq. 2.1 has bounded solutions.

Proposition 2.1 If $W: \mathbb{R} \rightarrow \mathbb{C}$ is a bounded solution of (2.1), the corresponding invariants ( $J, E)$ belong to the closure $\bar{D}$ of $D$ and the following properties hold:

(i) If $(J, E) \in D$ and $J \neq 0$, then the modulus $|W|$ and the derivative of the phase of $W$ are periodic with the same minimal period $T(J, E)>0$. If $(J, E) \in D$ and $J=0$, then $W$ is real-valued, up to a phase factor, and periodic with period $2 T(0, E)$.

(ii) If $(J, E) \in \partial D$, then either $W(x)=W_{q, \varphi}(x) \equiv\left(1-q^{2}\right)^{1 / 2} \mathrm{e}^{\mathrm{i}(q x+\varphi)}$ for some $q \in[-1,1]$ and some $\varphi \in[0,2 \pi]$, or $W$ is a homoclinic orbit connecting $W_{q, \varphi_{-}}$at $x=-\infty$ to $W_{q, \varphi_{+}}$at $x=+\infty$, for some $q^{2}<1 / 3$ and some $\varphi_{-}, \varphi_{+} \in[0,2 \pi]$.

Proof. Although the arguments are standard, we give a complete proof of Proposition 2.1 because it will serve as a basis for all subsequent developments in this section. Let $W: \mathbb{R} \rightarrow \mathbb{C}$ be a bounded solution of (2.1), and assume first that $J \neq 0$. Then $W(x)=r(x) \mathrm{e}^{\mathrm{i} \varphi(x)}$ and $E=\frac{1}{2} r_{x}^{2}+V_{J}(r)$, where $V_{J}$ is the "effective potential"

$$
V_{J}(r)=\frac{J^{2}}{2 r^{2}}+\frac{r^{2}}{2}-\frac{r^{4}}{4}, \quad r>0 .
$$

If $J^{2}>4 / 27$, then $V_{J}^{\prime}(r)<0$ for all $r>0$, hence (2.1) has no bounded solution in that case. Thus we must have $J^{2} \leq 4 / 27$. If $0<J^{2}<4 / 27$, we can parametrize $J$ in a unique way as

$$
J=q\left(1-q^{2}\right)=Q\left(1-Q^{2}\right), \quad \text { where } 0<q^{2}<1 / 3<Q^{2}<1 .
$$

Then $y^{3}-y+J=(y-q)(y-Q)(y+q+Q)$, hence in particular $J=q Q(q+Q)$. With this parametrization, it is easy to check that $V_{J}(r)$ has a (unique) local minimum at $r_{Q}=\left(1-Q^{2}\right)^{1 / 2}$ and a (unique) local maximum at $r_{q}=\left(1-q^{2}\right)^{1 / 2}$ (see Fig. 2). We define

$$
\begin{aligned}
& E_{-}(J)=V_{J}\left(\sqrt{1-Q^{2}}\right)=\frac{1}{4}\left(1-Q^{2}\right)\left(1+3 Q^{2}\right), \\
& E_{+}(J)=V_{J}\left(\sqrt{1-q^{2}}\right)=\frac{1}{4}\left(1-q^{2}\right)\left(1+3 q^{2}\right) .
\end{aligned}
$$

Since $W$ is a bounded solution of (2.1), we necessarily have $E_{-}(J) \leq E \leq E_{+}(J)$. This gives three possibilities: 
(i) If $E=E_{-}(J)$, then (up to a global phase factor) $W(x)=\left(1-Q^{2}\right)^{1 / 2} \mathrm{e}^{\mathrm{i} Q x}$, i.e. $W$ is a periodic solution with constant modulus.

(ii) If $E=E_{+}(J)$, then $|W|$ is either constant or homoclinic to $r_{q}$ as $x \rightarrow \pm \infty$. In the first case, $W(x)=\left(1-q^{2}\right)^{1 / 2} \mathrm{e}^{\mathrm{i} q x}$ (up to a phase factor). In the second case, up to a translation and a phase factor, we have the explicit formula

$$
W(x)=\left(2\left(q^{2}+c^{2} \tanh ^{2}(c x)\right)\right)^{1 / 2} \mathrm{e}^{\mathrm{i} q x+\mathrm{i} \arctan \left(\frac{c}{q} \tanh (c x)\right)}, \quad \text { where } c=\sqrt{\frac{1-3 q^{2}}{2}} .
$$

(iii) If $E_{-}(J)<E<E_{+}(J)$, the modulus $r=|W|$ and the phase derivative $\varphi_{x}=\operatorname{Im}\left(W_{x} / W\right)$ are periodic with the same period. If we denote by $r_{1}<r_{2}<r_{3}$ the three positive roots of $E-V_{J}(r)$ as in Fig. 2, this (minimal) period is

$$
T(J, E)=2 \int_{r_{1}(J, E)}^{r_{2}(J, E)} \frac{\mathrm{d} r}{\sqrt{2\left(E-V_{J}(r)\right)}} .
$$

Another important quantity is the increment of the phase $\varphi$ over a period of the modulus, namely

$$
\Phi(J, E)=2 \int_{r_{1}(J, E)}^{r_{2}(J, E)} \frac{J}{r^{2}} \frac{\mathrm{d} r}{\sqrt{2\left(E-V_{J}(r)\right)}} .
$$

Since in general $\Phi(J, E)$ is not a rational multiple of $\pi$, the solution $W(x)=r(x) \mathrm{e}^{\mathrm{i} \varphi(x)}$ of (2.1) is not periodic, but only quasi-periodic.

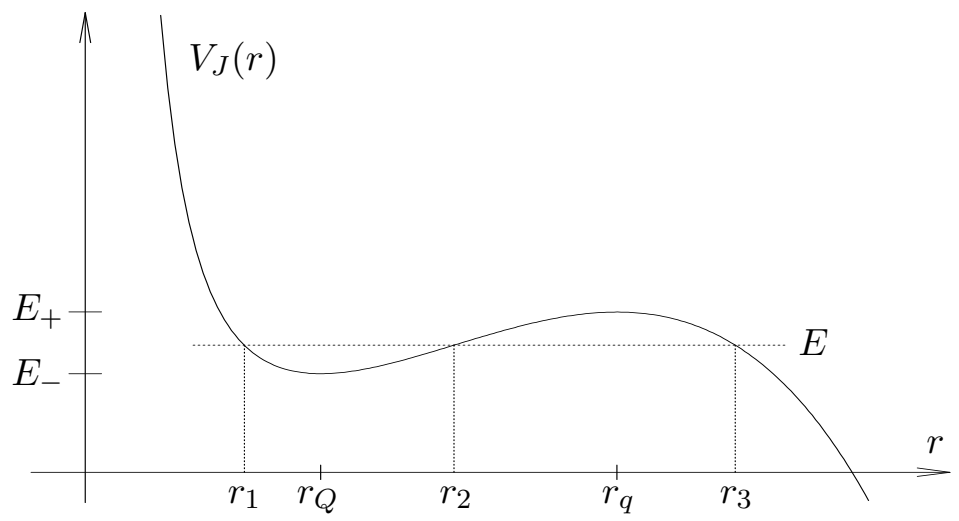

Fig. 2: When $0<J^{2}<4 / 27$, the effective potential $V_{J}$ has a local minimum at $r_{Q}=\left(1-Q^{2}\right)^{1 / 2}$ and a local maximum at $r_{q}=\left(1-q^{2}\right)^{1 / 2}$, where $q, Q$ are implicitely defined by $J=q\left(1-q^{2}\right)=Q\left(1-Q^{2}\right)$ and $q^{2}<1 / 3<Q^{2}$.

In the limiting case $J^{2}=4 / 27$, the effective potential $V_{J}$ is stricly decreasing over $\mathbb{R}_{+}$with an inflexion point at $r=(2 / 3)^{1 / 2}$. Since $W$ is bounded, we must have $E=E_{-}(J)=E_{+}(J)=1 / 3$, hence (up to a global phase) $W(x)=\left(1-q^{2}\right)^{1 / 2} \mathrm{e}^{\mathrm{i} q x}$ with $q=(1 / 3)^{1 / 2} \operatorname{sign}(J)$.

Finally, if $W$ is a solution of (2.1) with $J=0$, then (replacing $W(x)$ with $W(x) \mathrm{e}^{\mathrm{i} \varphi}$ for some $\varphi \in[0,2 \pi])$ we can assume that $W(x) \in \mathbb{R}$ for all $x \in \mathbb{R}$. Then $E=\frac{1}{2} r_{x}^{2}+V_{0}(r)$ and we have the 
same discussion as above with $E_{-}(0)=0$ and $E_{+}(0)=1 / 4$. If $E=0$, then $W \equiv 0$. If $E=1 / 4$, then either $W(x) \equiv \pm 1$ or $W(x)= \pm \tanh (x / \sqrt{2}$ ) (up to a translation). If $0<E<1 / 4$, then $W$ is periodic with half-period

$$
T(0, E)=2 \int_{0}^{r_{2}(0, E)} \frac{\mathrm{d} r}{\sqrt{2\left(E-V_{0}(r)\right)}}, \quad \text { where } \quad r_{2}(0, E)=(1-\sqrt{1-4 E})^{1 / 2} .
$$

Moreover, $W(x+T(0, E))=-W(x)$, hence $|W|$ is periodic with (minimal) period $T(0, E)$.

As we shall see at the end of this section, Proposition 2.1 implies the existence of a six-parameter family of quasi-periodic solutions of the nonlinear Schrödinger equation (1.1). Before doing that, we study in detail the properties of the period $T$ and the phase increment $\Phi$ defined in (2.5), (2.6), because these quantities play a crucial role in the stability analysis of the solutions of (2.1), both for the Schrödinger and the Ginzburg-Landau dynamics. We first give explicit formulas for $T$ and $\Phi$ (see also 9, 10]) which are convenient for analytical study and numerical approximation.

Lemma 2.2 Assume that $(J, E) \in D$ and denote by $0 \leq y_{1}<y_{2}<y_{3}$ the roots of the cubic polynomial $P(y)=y^{3}-2 y^{2}+4 E y-2 J^{2}$. Then

$$
T(J, E)=\sqrt{2} \int_{y_{1}}^{y_{2}} \frac{\mathrm{d} y}{\sqrt{\left(y-y_{1}\right)\left(y-y_{2}\right)\left(y-y_{3}\right)}}=2 \sqrt{2} \int_{0}^{\pi / 2} \frac{\mathrm{d} \varphi}{\sqrt{y_{3}-s(\varphi)}},
$$

where $s(\varphi)=y_{1} \cos ^{2}(\varphi)+y_{2} \sin ^{2}(\varphi)$. Similarly, if $J \neq 0$,

$$
\Phi(J, E)=\sqrt{2} \int_{y_{1}}^{y_{2}} \frac{J}{y} \frac{\mathrm{d} y}{\sqrt{\left(y-y_{1}\right)\left(y-y_{2}\right)\left(y-y_{3}\right)}}=2 \sqrt{2} \int_{0}^{\pi / 2} \frac{J}{s(\varphi)} \frac{\mathrm{d} \varphi}{\sqrt{y_{3}-s(\varphi)}} .
$$

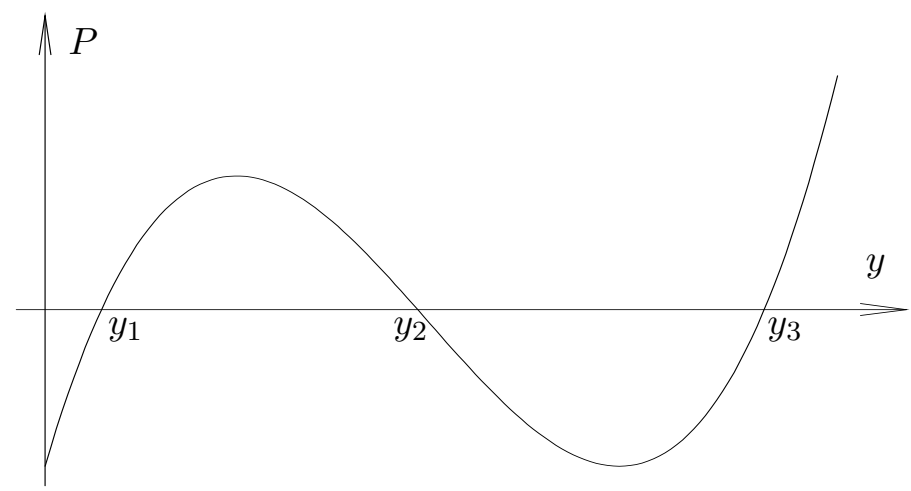

Fig. 3: The three roots of the cubic polynomial $P(y)=y^{3}-2 y^{2}+4 E y-2 J^{2}$ for $(J, E) \in D$.

Proof. Observe that $P\left(r^{2}\right)=4 r^{2}\left(E-V_{J}(r)\right)$, hence $P(y)$ has three nonnegative roots whenever $(J, E) \in D$ (see Fig. 2). Thus, using the change of variables $r=\sqrt{y}$ in (2.5), we obtain the first expression in (2.7). The last expression then follows by setting $y=s(\varphi)$, so that $\mathrm{d} y=$ $2 \sqrt{\left(y-y_{1}\right)\left(y_{2}-y\right)} \mathrm{d} \varphi$. Similarly, if $J \neq 0$, we obtain (2.8) from (2.6). 
It follows in particular from (2.7) that $T$ is a smooth function of $(J, E) \in D$. In contrast $\Phi$ cannot be extended to a continuous function over $D$, because (as is easily verified)

$$
\lim _{J \rightarrow 0^{ \pm}} \Phi(J, E)= \pm \pi, \quad \text { for } \quad 0<E<1 / 4
$$

This suggests to introduce the renormalized phase $\Psi: D \rightarrow \mathbb{R}$ defined by

$$
\Psi(J, E)=\left\{\begin{array}{clc}
\Phi(J, E)-\pi \operatorname{sign}(J) & \text { if } \quad J \neq 0 \\
0 & \text { if } \quad J=0
\end{array}\right.
$$

Lemma 2.3 For any $(J, E) \in D$, one has

$$
\Psi(J, E)=2 \sqrt{2} J \int_{0}^{\pi / 2} \frac{\mathrm{d} \varphi}{\sqrt{y_{3}} \sqrt{y_{3}-s(\varphi)}\left(\sqrt{y_{3}}+\sqrt{y_{3}-s(\varphi)}\right)} .
$$

In particular, $\Psi: D \rightarrow \mathbb{R}$ is a smooth function.

Proof. The integral in (2.10) is a smooth function of $(J, E) \in D$, so it is sufficient to establish (2.10) for $J \neq 0$. In that case, we remark that

$$
2 \sqrt{2} \int_{0}^{\pi / 2} \frac{J}{s(\varphi)} \frac{1}{\sqrt{y_{3}}} \mathrm{~d} \varphi=\frac{2 \sqrt{2}}{\sqrt{y_{3}}}\left(\frac{\pi}{2} \frac{J}{\sqrt{y_{1} y_{2}}}\right)=\frac{\pi \sqrt{2} J}{\sqrt{y_{1} y_{2} y_{3}}}=\pi \operatorname{sign}(J),
$$

because $y_{1} y_{2} y_{3}=2 J^{2}$. Thus, using (2.8) and (2.9), we obtain

$$
\begin{aligned}
\Psi(J, E) & =2 \sqrt{2} \int_{0}^{\pi / 2} \frac{J}{s(\varphi)}\left(\frac{1}{\sqrt{y_{3}-s(\varphi)}}-\frac{1}{\sqrt{y_{3}}}\right) \mathrm{d} \varphi \\
& =2 \sqrt{2} J \int_{0}^{\pi / 2} \frac{\mathrm{d} \varphi}{\sqrt{y_{3}} \sqrt{y_{3}-s(\varphi)}\left(\sqrt{y_{3}}+\sqrt{y_{3}-s(\varphi)}\right)},
\end{aligned}
$$

which is the desired formula.

We next study the monotonicity properties of $T$ and $\Psi$. Since $T(J, E)$ is an even and $\Psi(J, E)$ an odd function of $J$, we may restrict ourselves to the half-domain $D_{+}=\{(J, E) \in D \mid J>0\}$.

\section{Proposition 2.4}

i) $\frac{\partial T}{\partial E}(J, E)>0$ for all $(J, E) \in D$.

ii) $\frac{\partial \Psi}{\partial E}(J, E) \equiv \frac{\partial \Phi}{\partial E}(J, E)=-\frac{\partial T}{\partial J}(J, E)>0$ for all $(J, E) \in D_{+}$.

Proof. The monotonicity of the period $T$ with respect to the energy $E$ has been established in [9]. We give here a different argument, which is also a preparation for the proof of Proposition [2.6 below. Let $(J, E) \in D$. Since $y_{1}, y_{2}, y_{3}$ are solutions of the cubic equation $y^{3}-2 y^{2}+4 E y-2 J^{2}=0$, we have $y_{1}+y_{2}+y_{3}=2$ and

$$
\frac{\partial y_{i}}{\partial E}=-\frac{4 y_{i}}{3 y_{i}^{2}-4 y_{i}+4 E}, \quad \frac{\partial y_{i}}{\partial J}=\frac{4 J}{3 y_{i}^{2}-4 y_{i}+4 E}, \quad i=1,2,3 .
$$


In particular,

$$
\frac{\partial y_{1}}{\partial E}<0, \quad \frac{\partial y_{2}}{\partial E}>0, \quad \frac{\partial y_{1}}{\partial E}+\frac{\partial y_{2}}{\partial E}=-\frac{\partial y_{3}}{\partial E}>0
$$

because $P^{\prime}\left(y_{1}\right)>0, P^{\prime}\left(y_{2}\right)<0, P^{\prime}\left(y_{3}\right)>0$ (see Fig. 3). Similarly, if $J>0$,

$$
\frac{\partial y_{1}}{\partial J}>0, \quad \frac{\partial y_{2}}{\partial J}<0, \quad \frac{\partial y_{1}}{\partial J}+\frac{\partial y_{2}}{\partial J}=-\frac{\partial y_{3}}{\partial J}<0 .
$$

On the other hand, differentiating (2.7) with respect to $E$ and $J$, we find

$$
\frac{\partial T}{\partial E}=A_{1} \frac{\partial y_{1}}{\partial E}+A_{2} \frac{\partial y_{2}}{\partial E}, \quad \frac{\partial T}{\partial J}=A_{1} \frac{\partial y_{1}}{\partial J}+A_{2} \frac{\partial y_{2}}{\partial J}
$$

where

$$
A_{1}=\sqrt{2} \int_{0}^{\pi / 2} \frac{1+\cos ^{2}(\varphi)}{\left(y_{3}-s(\varphi)\right)^{3 / 2}} \mathrm{~d} \varphi, \quad A_{2}=\sqrt{2} \int_{0}^{\pi / 2} \frac{1+\sin ^{2}(\varphi)}{\left(y_{3}-s(\varphi)\right)^{3 / 2}} \mathrm{~d} \varphi .
$$

The crucial observation is:

$$
A_{2}-A_{1}=\sqrt{2} \int_{0}^{\pi / 2} \frac{\sin ^{2}(\varphi)-\cos ^{2}(\varphi)}{\left(y_{3}-s(\varphi)\right)^{3 / 2}} \mathrm{~d} \varphi>0 .
$$

Indeed, this inequality follows from Lemma 2.5 below, with $I=[0, \pi / 2], \mathrm{d} \mu=(2 / \pi) \mathrm{d} \varphi, f(\varphi)=$ $\sin ^{2}(\varphi)-\cos ^{2}(\varphi)$ and $g(\varphi)=\left(y_{3}-s(\varphi)\right)^{-3 / 2}$ (remark that $f, g$ are strictly increasing, and that $\int_{0}^{\pi / 2} f(\varphi) \mathrm{d} \varphi=0$.) Thus $A_{2}>A_{1}>0$. We conclude that

$$
\frac{\partial T}{\partial E}=\left(A_{2}-A_{1}\right) \frac{\partial y_{2}}{\partial E}+A_{1}\left(\frac{\partial y_{1}}{\partial E}+\frac{\partial y_{2}}{\partial E}\right)>0 .
$$

Similarly, if $J>0$,

$$
\frac{\partial T}{\partial J}=\left(A_{2}-A_{1}\right) \frac{\partial y_{2}}{\partial J}+A_{1}\left(\frac{\partial y_{1}}{\partial J}+\frac{\partial y_{2}}{\partial J}\right)<0 .
$$

Finally, the relation $\partial T / \partial J=-\partial \Phi / \partial E$ is a consequence of the following standard observation [5]: if $\mathcal{A}: D \rightarrow \mathbb{R}$ is the action functional defined by

$$
\mathcal{A}(J, E)=2 \int_{r_{1}(J, E)}^{r_{2}(J, E)} \sqrt{2\left(E-V_{J}(r)\right)} \mathrm{d} r
$$

then a direct calculation shows that $T=\partial \mathcal{A} / \partial E$ and $\Phi=-\partial \mathcal{A} / \partial J$, and the result follows.

The following elementary result will be used several times in this paper:

Lemma 2.5 Let $\mu$ be a (Borel) probability measure on some interval $I \subset \mathbb{R}$, and let $f, g: I \rightarrow \mathbb{R}$ be bounded and measurable functions. Then

$$
\int_{I} f(x) g(x) \mathrm{d} \mu-\left(\int_{I} f(x) \mathrm{d} \mu\right)\left(\int_{I} g(x) \mathrm{d} \mu\right)=\frac{1}{2} \int_{I \times I}(f(x)-f(y))(g(x)-g(y)) \mathrm{d} \mu_{x} \mathrm{~d} \mu_{y} .
$$

In particular, if both $f$ and $g$ are strictly increasing or strictly decreasing, and if the support of $\mu$ is not reduced to a single point, then

$$
\int_{I} f(x) g(x) \mathrm{d} \mu>\left(\int_{I} f(x) \mathrm{d} \mu\right)\left(\int_{I} g(x) \mathrm{d} \mu\right) .
$$


We now establish an important non-degeneracy property of system (2.1). For any $(J, E) \in D$, let

$$
\Delta(J, E)=\operatorname{det}\left(\begin{array}{ll}
\frac{\partial T}{\partial E} & \frac{\partial \Psi}{\partial E} \\
\frac{\partial T}{\partial J} & \frac{\partial \Psi}{\partial J}
\end{array}\right)(J, E) .
$$

Proposition 2.6 For all $(J, E) \in D$ we have $\Delta(J, E)>0$.

\section{Remarks}

1. Proposition 2.6 shows that the integrable Hamiltonian system (2.1) is non-degenerate in the sense of KAM theory. Indeed, by Liouville's theorem, we can express (at least locally) the energy $E$ of the system in terms of the action variables $J$ and $\mathcal{A}$, where $\mathcal{A}$ is defined in (2.17). If we denote $E=H(J, \mathcal{A})$, a direct calculation [5] shows that

$$
\Delta=-T^{4} \operatorname{det}\left(\begin{array}{cc}
\frac{\partial^{2} H}{\partial \mathcal{A}^{2}} & \frac{\partial^{2} H}{\partial \mathcal{A} \partial J} \\
\frac{\partial^{2} H}{\partial J \partial \mathcal{A}} & \frac{\partial^{2} H}{\partial J^{2}}
\end{array}\right),
$$

hence the KAM determinant (the Hessian of $H$ ) is always negative.

2. By Proposition 2.4 it is clear that $\Delta>0$ whenever $\partial \Psi / \partial J>0$. Unfortunately, the latter inequality is not true for all $(J, E) \in D$.

Proof. To evaluate $\Delta$ we have to compute the derivatives of the renormalized phase $\Psi$ with respect to $E$ and $J$. Using (2.10), we obtain after straightforward calculations:

$$
\frac{\partial \Psi}{\partial E}=B_{1} J \frac{\partial y_{1}}{\partial E}+B_{2} J \frac{\partial y_{2}}{\partial E}, \quad \frac{\partial \Psi}{\partial J}=B_{1} J \frac{\partial y_{1}}{\partial J}+B_{2} J \frac{\partial y_{2}}{\partial J}+B_{3}
$$

where

$$
\begin{aligned}
& B_{1}= \sqrt{2} \int_{0}^{\pi / 2}\left\{\frac{1}{B_{2} y_{3}^{3 / 2}\left(y_{3}-s(\varphi)\right)^{1 / 2}} \frac{2 \sqrt{y_{3}}+\sqrt{y_{3}-s(\varphi)}}{\left(\sqrt{y_{3}}+\sqrt{y_{3}-s(\varphi)}\right)^{2}}+\right. \\
&\left.\frac{1}{y_{3}^{1 / 2}\left(y_{3}-s(\varphi)\right)^{3 / 2}} \frac{\sqrt{y_{3}}+2 \sqrt{y_{3}-s(\varphi)}}{\left(\sqrt{y_{3}}+\sqrt{y_{3}-s(\varphi)}\right)^{2}}\left(1+\begin{array}{c}
\cos ^{2}(\varphi) \\
\sin ^{2}(\varphi)
\end{array}\right)\right\} \mathrm{d} \varphi, \\
& B_{3}=2 \sqrt{2} \int_{0}^{\pi / 2} \frac{1}{\sqrt{y_{3}} \sqrt{y_{3}-s(\varphi)}\left(\sqrt{y_{3}}+\sqrt{y_{3}-s(\varphi)}\right)} \mathrm{d} \varphi=\frac{\Psi}{J} .
\end{aligned}
$$

(Here the formula for $B_{1}$ should be read with $\cos ^{2}(\varphi)$ in the right-hand side, and $B_{2}$ with $\sin ^{2}(\varphi)$; both expressions are otherwise identical.) Using (2.14), (2.19) together with the identities

$$
J \frac{\partial y_{i}}{\partial E}+y_{i} \frac{\partial y_{i}}{\partial J}=0, \quad i=1,2,3
$$

which follow from (2.11), we find

$$
\begin{aligned}
\Delta(J, E)= & \left(A_{1} \frac{\partial y_{1}}{\partial E}+A_{2} \frac{\partial y_{2}}{\partial E}\right)\left(B_{1} J \frac{\partial y_{1}}{\partial J}+B_{2} J \frac{\partial y_{2}}{\partial J}+B_{3}\right) \\
& -\left(A_{1} \frac{\partial y_{1}}{\partial J}+A_{2} \frac{\partial y_{2}}{\partial J}\right)\left(B_{1} J \frac{\partial y_{1}}{\partial E}+B_{2} J \frac{\partial y_{2}}{\partial E}\right) \\
= & J\left(A_{1} B_{2}-A_{2} B_{1}\right)\left(\frac{\partial y_{1}}{\partial E} \frac{\partial y_{2}}{\partial J}-\frac{\partial y_{2}}{\partial E} \frac{\partial y_{1}}{\partial J}\right)+B_{3} \frac{\partial T}{\partial E} \\
= & \left(A_{1} B_{2}-A_{2} B_{1}\right)\left(y_{2}-y_{1}\right) \frac{\partial y_{1}}{\partial J} \frac{\partial y_{2}}{\partial J}+B_{3} \frac{\partial T}{\partial E} .
\end{aligned}
$$


Since $\partial T / \partial E>0, B_{3}>0, y_{2}-y_{1}>0$ and $\left(\partial y_{1} / \partial J\right)\left(\partial y_{2} / \partial J\right)<0$, it is sufficient to prove that $\Delta_{1} \stackrel{\text { def }}{=} A_{2} B_{1}-A_{1} B_{2}>0$.

To achieve this goal, we set $\sigma(\varphi)=\left(1-y_{3}^{-1} s(\varphi)\right)^{1 / 2}$ and we observe that $A_{i}=\sqrt{2} y_{3}^{-3 / 2} \mathbf{A}_{i}$, $B_{i}=\sqrt{2} y_{3}^{-5 / 2} \mathbf{B}_{i}$, for $i=1,2$, where

$$
\begin{aligned}
& \mathbf{A}_{1}=\int_{0}^{\pi / 2} \frac{1+\cos ^{2}(\varphi)}{\sigma(\varphi)^{3}} \mathrm{~d} \varphi, \quad \mathbf{A}_{2}=\int_{0}^{\pi / 2} \frac{1+\sin ^{2}(\varphi)}{\sigma(\varphi)^{3}} \mathrm{~d} \varphi, \\
& \mathbf{B}_{1}=\int_{0}^{\pi / 2}\left\{\frac{2+\sigma(\varphi)}{\sigma(\varphi)(1+\sigma(\varphi))^{2}}+\frac{1+2 \sigma(\varphi)}{\sigma(\varphi)^{3}(1+\sigma(\varphi))^{2}}\left(1+\begin{array}{c}
\cos ^{2}(\varphi) \\
\sin ^{2}(\varphi)
\end{array}\right)\right\} \mathrm{d} \varphi .
\end{aligned}
$$

Thus $\Delta_{1}=2 y_{3}^{-4} \Delta_{2}$, where $\Delta_{2}=\mathbf{A}_{2} \mathbf{B}_{1}-\mathbf{A}_{1} \mathbf{B}_{2}$. To prove that $\Delta_{2}>0$, we remark that

$$
\frac{1+2 \sigma}{\sigma^{3}(1+\sigma)^{2}}=\frac{1}{\sigma^{3}}-\frac{1}{\sigma(1+\sigma)^{2}}, \quad \text { and } \quad \frac{2+\sigma}{\sigma(1+\sigma)^{2}}-\frac{1+\cos ^{2}(\varphi)}{\sigma(1+\sigma)^{2}}=\frac{\sigma+\sin ^{2}(\varphi)}{\sigma(1+\sigma)^{2}} \text {. }
$$

It follows that $\mathbf{B}_{1}=\mathbf{A}_{1}+\tilde{\mathbf{B}}_{1}$ and $\mathbf{B}_{2}=\mathbf{A}_{2}+\tilde{\mathbf{B}}_{2}$, where

$$
\tilde{\mathbf{B}}_{1}=\int_{0}^{\pi / 2} \frac{\sigma(\varphi)+\sin ^{2}(\varphi)}{\sigma(\varphi)(1+\sigma(\varphi))^{2}} \mathrm{~d} \varphi, \quad \tilde{\mathbf{B}}_{2}=\int_{0}^{\pi / 2} \frac{\sigma(\varphi)+\cos ^{2}(\varphi)}{\sigma(\varphi)(1+\sigma(\varphi))^{2}} \mathrm{~d} \varphi .
$$

Now, we know from (2.15) that $\mathbf{A}_{2}>\mathbf{A}_{1}>0$, and the same argument (using Lemma 2.5) shows that $\tilde{\mathbf{B}}_{1}>\tilde{\mathbf{B}}_{2}>0$. Thus

$$
\Delta_{2}=\mathbf{A}_{2} \mathbf{B}_{1}-\mathbf{A}_{1} \mathbf{B}_{2}=\mathbf{A}_{2} \tilde{\mathbf{B}}_{1}-\mathbf{A}_{1} \tilde{\mathbf{B}}_{2}>0
$$

and the proof is complete.

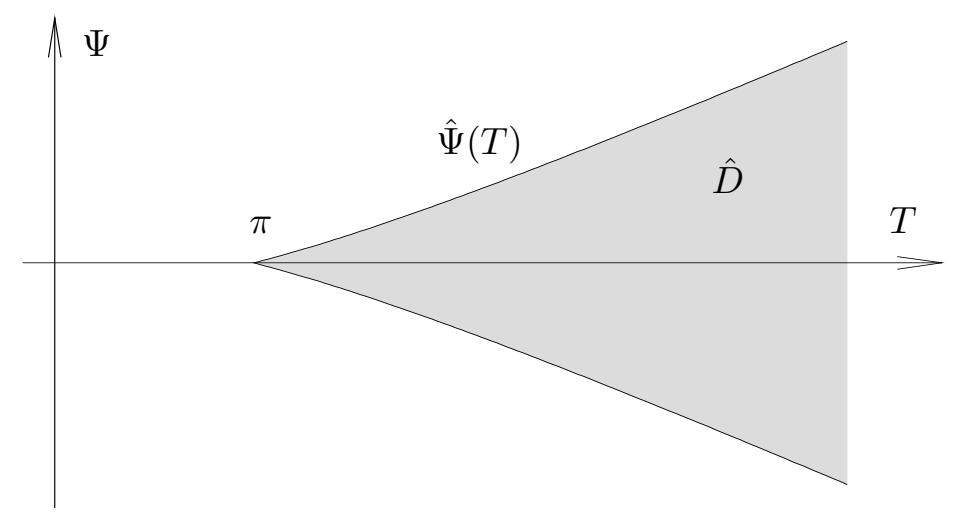

Fig. 4: The region $\hat{D} \subset \mathbb{R}^{2}$ consisting of all possible values of the pair $(T, \Psi)$.

Propositions 2.4 and 2.6 imply that the quasi-periodic solutions of (2.1) can be parametrized by the period $T$ and the renormalized phase $\Psi$, instead of the angular momentum $J$ and the energy $E$. Indeed, let

$$
\hat{D}=\left\{(T, \Psi) \in \mathbb{R}^{2}|T>\pi,| \Psi \mid<\hat{\Psi}(T)\right\}, \quad \text { where } \quad \hat{\Psi}(T)=\left(\frac{T^{2}+2 \pi^{2}}{3}\right)^{1 / 2}-\pi .
$$


Proposition 2.7 The map $(T, \Psi): D \rightarrow \hat{D}$ is a smooth diffeomorphism.

Proof. Let $J=Q\left(1-Q^{2}\right)$, where $1 / 3<Q^{2} \leq 1$. Using (2.7) it is straightforward to verify that

$$
\lim _{E \rightarrow E_{-}(J)} T(J, E)=\frac{\pi \sqrt{2}}{\sqrt{3 Q^{2}-1}} \equiv \frac{\pi}{\left(1-3 E_{-}(J)\right)^{1 / 4}}, \quad \lim _{E \rightarrow E_{+}(J)} T(J, E)=+\infty .
$$

Similarly, if $1 / \sqrt{3}<Q<1$ (so that $J>0$ ), we deduce from (2.8) that

$$
\lim _{E \rightarrow E_{-}(J)} \Phi(J, E)=\frac{\pi \sqrt{2} Q}{\sqrt{3 Q^{2}-1}}, \quad \lim _{E \rightarrow E_{+}(J)} \Phi(J, E)=+\infty .
$$

Since $\partial T / \partial E>0$ by Proposition 2.4 it follows from (2.24) that the range of the map $T: D \rightarrow \mathbb{R}$ is exactly the interval $(\pi,+\infty)$. Fix $T_{0}>\pi$ and let $\Sigma=\left\{(J, E) \in D \mid T(J, E)=T_{0}\right\}$. By the Implicit Function Theorem, $\Sigma$ is a smooth curve in $D$ which can be represented as a graph over the $J$-axis. Moreover, we know that $\Sigma$ connects the boundary points $\left(-J_{0}, E_{-}\left(J_{0}\right)\right)$ and $\left(J_{0}, E_{-}\left(J_{0}\right)\right)$, where $J_{0}=Q_{0}\left(1-Q_{0}^{2}\right)$ and $Q_{0} \in(1 / \sqrt{3}, 1)$ is determined by the relation

$$
\frac{\pi \sqrt{2}}{\sqrt{3 Q_{0}^{2}-1}}=T_{0}
$$

(see (2.24) ). Now, Proposition 2.6 implies that the restriction of $\Psi$ to the curve $\Sigma$ is a strictly increasing function of $J$, because

$$
\left.\frac{\mathrm{d}}{\mathrm{d} J} \Psi\right|_{\Sigma}=\left(\frac{\partial T}{\partial E}\right)^{-1}\left(\frac{\partial T}{\partial E} \frac{\partial \Psi}{\partial J}-\frac{\partial T}{\partial J} \frac{\partial \Psi}{\partial E}\right)>0
$$

Thus $\Psi$ varies from $-\Psi_{0}$ to $\Psi_{0}$ on the curve $\Sigma$, where by (2.25)

$$
\Psi_{0}=\frac{\pi \sqrt{2} Q_{0}}{\sqrt{3 Q_{0}^{2}-1}}-\pi=\left(\frac{T_{0}^{2}+2 \pi^{2}}{3}\right)^{1 / 2}-\pi \equiv \hat{\Psi}\left(T_{0}\right) .
$$

This proves that $(T, \Psi): D \rightarrow \hat{D}$ is onto, and the monotonicity properties established in Proposition 2.4 imply that $(T, \Psi)$ is also one-to-one.

To conclude this section, we briefly verify that Proposition 2.1 implies the existence of periodic waves of the NLS equation of the form (1.2). Fix $(J, E) \in D$, and let $W: \mathbb{R} \rightarrow \mathbb{C}$ be a bounded solution of (2.1) satisfying (2.2). (The proof of Proposition 2.1 shows that this solution is unique up to a translation and a phase factor. If needed, we can specify a particular solution by imposing for instance $W(0)=r_{2}, W^{\prime}(0)=\mathrm{i} J / r_{2}$, where $r_{2}>0$ is as in Fig. 2.) We now set

$$
W(x)=\mathrm{e}^{\mathrm{i} \ell x} P(k x), \quad x \in \mathbb{R},
$$

where

$$
k=\frac{\pi}{T(J, E)}, \quad \text { and } \quad \ell=\frac{\Psi(J, E)}{T(J, E)} .
$$

As $x \mapsto|W(x)|$ is periodic with minimal period $T(J, E)$, it is clear that $y \mapsto|P(y)|$ is periodic with minimal period $\pi$. Moreover, since $W(x+T)=\mathrm{e}^{\mathrm{i} \Phi} W(x)$ by definition of $\Phi(J, E)$, we also have $P(y+\pi)=-P(y)$ for all $y \in \mathbb{R}$, hence $P$ is $2 \pi$-periodic. Thus $U(x, t)=\mathrm{e}^{-\mathrm{i} t} W(x)=\mathrm{e}^{\mathrm{i}(\ell x-t)} P(k x)$ is a quasi-periodic solution of (1.1) of the form (1.2), with $\omega=1$ and $c=0$. 
Remark 2.8 Using the continuous symmetries of the NLS equation, we can produce for each pair $(J, E) \in D$ a four-parameter family of periodic waves:

$$
U_{c, \lambda, \varphi, \xi}(x, t)=\lambda \mathrm{e}^{\mathrm{i}\left(p_{c, \lambda} x-\omega_{c, \lambda} t-\varphi\right)} P(k \lambda(x-c t)+\xi),
$$

where $c \in \mathbb{R}, \lambda>0, \varphi, \xi \in[0,2 \pi]$, and $p_{c, \lambda}=\lambda \ell+c / 2, \omega_{c, \lambda}=\lambda^{2}+c \lambda \ell+c^{2} / 4$. Taking into account the parameters $J, E$, we obtain altogether a six-parameter family of periodic waves of (1.1).

The representation (2.26) is well-adapted to understand the connection between the bounded solutions $W$ of (2.1) and the periodic waves of the NLS equation, especially in the case of the cnoidal waves for which $J=\ell=0$. However it is more convenient for our purposes to write the solution $W$ of (2.1) in the alternative form

$$
W(x)=\mathrm{e}^{\mathrm{i}(\ell+k) x} Q^{+}(2 k x)=\mathrm{e}^{\mathrm{i}(\ell-k) x} Q^{-}(2 k x), \quad x \in \mathbb{R},
$$

where $Q^{ \pm}(z)=\mathrm{e}^{\mp \mathrm{i} z / 2} P(z / 2)$. By construction, $Q^{ \pm}$and $\left|Q^{ \pm}\right|$are now periodic functions with the same minimal period $2 \pi$. This property facilitates the description of the special class of perturbations that we use for the stability analysis, see the statement of Theorem 1 and the remarks thereafter. The representation (2.28) is also very natural for solutions which are close to plane waves: in such a situation, either $Q^{+}$or $Q^{-}$is close to a constant, depending on the sign of $J$. This follows from the fact that $\ell+k=\Phi / T$ if $J>0$ and $\ell-k=\Phi / T$ if $J<0$.

\section{Orbital stability}

Our aim in this section is to show that the periodic waves of the defocusing nonlinear Schrödinger equation (1.1) with $\gamma=-1$ are stable within the class of solutions which have the same period $T$ and the same Floquet multiplier $\mathrm{e}^{\mathrm{i} \Phi}$. Given $(J, E) \in D$, where $D \subset \mathbb{R}^{2}$ is the parameter domain (2.3), we consider the periodic wave $U_{J, E}(x, t)=\mathrm{e}^{-\mathrm{i} t} W_{J, E}(x)$, where $W_{J, E}$ is the unique solution of (2.1) with initial data $W_{J, E}(0)=r_{2}, W_{J, E}^{\prime}(0)=\mathrm{i} J / r_{2}$, and $r_{2}>0$ is as in Fig. 2. In particular, $W_{J, E}$ satisfies (2.2). Let $p=k+\ell$, where $k \equiv k_{J, E}$ and $\ell \equiv \ell_{J, E}$ are defined by (2.27). As in (2.28), we set $W_{J, E}(x)=\mathrm{e}^{\mathrm{i} p x} Q_{J, E}(2 k x)$ and we recall that $Q_{J, E}(z)$ is a $2 \pi$-periodic function of $z$.

To study the stability of the periodic wave $U_{J, E}(x, t)$, we consider solutions of (1.1) of the form

$$
U(x, t)=\mathrm{e}^{\mathrm{i}(p x-t)} Q(2 k x, t)
$$

where $Q(z, t)$ satisfies the evolution equation

$$
\mathrm{i} Q_{t}+4 \mathrm{i} p k Q_{z}+4 k^{2} Q_{z z}+\left(1-p^{2}\right) Q-|Q|^{2} Q=0 .
$$

By construction, $Q_{J, E}(z)$ is now a stationary solution of (3.2) and our goal is to show that this equilibrium is stable with respect to $2 \pi$-periodic perturbations. We thus introduce the function space

$$
X=H_{\mathrm{per}}^{1}([0,2 \pi], \mathbb{C})=\left\{u \in H_{\mathrm{loc}}^{1}(\mathbb{R}, \mathbb{C}) \mid u(z)=u(z+2 \pi) \text { for all } z \in \mathbb{R}\right\}
$$


which is viewed as a real Hilbert space equipped with the scalar product

$$
(u, v)_{X}=\operatorname{Re} \int_{0}^{2 \pi}\left(u(z) \bar{v}(z)+u_{z}(z) \bar{v}_{z}(z)\right) \mathrm{d} z, \quad u, v \in X
$$

As usual, the dual space $X^{*}$ will be identified with $H_{\text {per }}^{-1}([0,2 \pi], \mathbb{C})$ through the pairing

$$
\langle u, v\rangle=\operatorname{Re} \int_{0}^{2 \pi} u(z) \bar{v}(z) \mathrm{d} z, \quad u \in X^{*}, \quad v \in X .
$$

It is well-known that the Cauchy problem for (3.2) is globally well-posed in the space $X$ (see [8, 13, 14, 17]). Moreover, the evolution defined by (3.2) on $X$ is invariant under the action of a two-parameter group of isometries: the space translations and the phase rotations. The symmetry group $G$ is thus the two-dimensional torus $\mathbb{T}^{2}=(\mathbb{R} / 2 \pi \mathbb{Z})^{2}$ which acts on $X$ through the unitary representation $\mathcal{R}$ defined by

$$
\left(\mathcal{R}_{(\varphi, \xi)} u\right)(z)=\mathrm{e}^{-\mathrm{i} \varphi} u(z+\xi), \quad u \in X, \quad(\varphi, \xi) \in G
$$

In view of these symmetries, it is natural to introduce the semi-distance $\rho$ on $X$ defined by

$$
\rho(u, v)=\inf _{(\varphi, \xi) \in G}\left\|u-\mathcal{R}_{(\varphi, \xi)} v\right\|_{X}, \quad u, v \in X
$$

The main result of this section is the following reformulation of Theorem 1

Proposition 3.1 Given $(J, E) \in D$, there exist $C_{0}>0$ and $\epsilon_{0}>0$ such that, if $Q_{0} \in X$ satisfies $\rho\left(Q_{0}, Q_{J, E}\right) \leq \epsilon$ for some $\epsilon \leq \epsilon_{0}$, then the solution $Q(z, t)$ of (3.2) with initial data $Q_{0}$ satisfies $\rho\left(Q(\cdot, t), Q_{J, E}\right) \leq C_{0} \epsilon$ for all $t \in \mathbb{R}$.

To prove Proposition 3.1. we follow the general approach of Grillakis, Shatah, and Strauss [15, 16. We first observe that Eq.(3.2) inherits from the original NLS equation several conserved quantities:

$$
\begin{aligned}
& N(Q)=\frac{1}{2} \int_{0}^{2 \pi}|Q(z)|^{2} \mathrm{~d} z \\
& M(Q)=\frac{\mathrm{i}}{2} \int_{0}^{2 \pi} \bar{Q}(z) Q_{z}(z) \mathrm{d} z \\
& \mathcal{E}(Q)=\int_{0}^{2 \pi}\left(2 k^{2}\left|Q_{z}(z)\right|^{2}+\frac{1}{4}|Q(z)|^{4}\right) \mathrm{d} z,
\end{aligned}
$$

which will be referred to as the charge, the momentum, and the energy, respectively. The charge $N$ is conserved due to the phase invariance of (3.2), the momentum $M$ due to the translation invariance, and the energy $\mathcal{E}$ because (3.2) is autonomous. Clearly $N, M$, and $\mathcal{E}$ are smooth real functions on $X$. Their first order derivatives are therefore smooth maps from $X$ into $X^{*}$ :

$$
N^{\prime}(Q)=Q, \quad M^{\prime}(Q)=\mathrm{i} Q_{z}, \quad \mathcal{E}^{\prime}(Q)=-4 k^{2} Q_{z z}+|Q|^{2} Q
$$


Similarly, the second order derivatives are smooth maps from $X$ into $\mathcal{L}\left(X, X^{*}\right)$, the space of all bounded linear operators from $X$ into $X^{*}$ :

$$
N^{\prime \prime}(Q)=\mathbf{1}, \quad M^{\prime \prime}(Q)=\mathrm{i} \partial_{z}, \quad \mathcal{E}^{\prime \prime}(Q)=-4 k^{2} \partial_{z z}+|Q|^{2}+2 Q \otimes Q
$$

where $\langle(Q \otimes Q) u, v\rangle=\int_{0}^{2 \pi} \operatorname{Re}(Q \bar{u}) \operatorname{Re}(Q \bar{v}) \mathrm{d} z$ for all $u, v \in X$.

By construction, the periodic wave profile $Q_{J, E}$ is a critical point of the modified energy

$$
\mathcal{E}_{J, E}(Q)=\mathcal{E}(Q)-\left(1-p^{2}\right) N(Q)-4 p k M(Q)
$$

namely $\mathcal{E}_{J, E}^{\prime}\left(Q_{J, E}\right)=0$. To determine the nature of this critical point, we consider the second variation

$$
H_{J, E}=\mathcal{E}_{J, E}^{\prime \prime}\left(Q_{J, E}\right)=-4 k^{2} \partial_{z z}-4 \mathrm{i} p k \partial_{z}-\left(1-p^{2}\right)+\left|Q_{J, E}\right|^{2}+2 Q_{J, E} \otimes Q_{J, E} .
$$

Since $X$ is a real Hilbert space, it is natural to decompose its elements (which are complex functions) into real and imaginary parts, in which case we obtain the matrix operator

$$
\hat{H}_{J, E}=\left(\begin{array}{cc}
-4 k^{2} \partial_{z z}-\left(1-p^{2}\right)+3 R_{J, E}^{2}+I_{J, E}^{2} & 4 p k \partial_{z}+2 R_{J, E} I_{J, E} \\
-4 p k \partial_{z}+2 R_{J, E} I_{J, E} & -4 k^{2} \partial_{z z}-\left(1-p^{2}\right)+R_{J, E}^{2}+3 I_{J, E}^{2}
\end{array}\right),
$$

where $Q_{J, E}=R_{J, E}+\mathrm{i} I_{J, E}$. As is easily verified, $H_{J, E}$ is a self-adjoint operator in $X_{0}:=$ $L_{\text {per }}^{2}([0,2 \pi], \mathbb{C})$ with compact resolvent, and $H_{J, E}$ is bounded from below. According to [15, 16], a crucial information is the number of negative eigenvalues of this operator.

Proposition 3.2 For any $(J, E) \in D$, the operator $H_{J, E}$ acting on $X_{0}$ has a simple negative eigenvalue, a double eigenvalue at zero, and the rest of the spectrum is strictly positive.

Proof. Since Eq.(3.2) is invariant under the action of $G$, we know that $\mathcal{R}_{(\varphi, \xi)} Q_{J, E}$ is a stationary solution for all $(\varphi, \xi) \in G$. Differentiating with respect to $\varphi$ and $\xi$ at $(\varphi, \xi)=(0,0)$, we obtain $H_{J, E} Q_{J, E}^{\prime}=H_{J, E}\left(\mathrm{i} Q_{J, E}\right)=0$. It is clear that the functions $Q_{J, E}^{\prime}$ and $\mathrm{i} Q_{J, E}$ are linearly independent in the domain $X_{2}:=H_{\text {per }}^{2}\left([0,2 \pi], \mathbb{C}\right.$ ) of $H_{J, E}$ (otherwise $W_{J, E}(x)=\mathrm{e}^{\mathrm{i} p x} Q_{J, E}(2 k x)$ would be a plane wave, contradicting the assumption $(J, E) \in D)$. Thus we see that zero is an eigenvalue of $H_{J, E}$ of multiplicity at least two.

Following 9], we next show that the multiplicity of zero as an eigenvalue of $H_{J, E}$ in $X_{0}$ is always exactly two. The idea is to produce two other solutions of the differential equation $H_{J, E} Q=0$ by differentiating the profile $Q_{J, E}$ with respect to $J$ and $E$, and to verify that none of these solutions belongs to the domain $X_{2}$. Since the kernel $K=\left\{Q \in C^{2}(\mathbb{R}, \mathbb{C}) \mid H_{J, E} Q=0\right\}$ is a four-dimensional (real) vector space, it will follow that $K \cap X_{2}=\operatorname{span}\left\{Q_{J, E}^{\prime}, \mathrm{i} Q_{J, E}\right\}$, which is the desired result.

However, the profile $Q_{J, E}$ is a stationary solution of the equation (3.2) which has coefficients depending upon $J$ and $E$, so that we cannot find the two solutions of $H_{J, E} Q=0$ just by differentiating $Q_{J, E}$ with respect to $J$ and $E$. Instead, we start with the quasi-periodic solution $W_{J, E}(x)=\mathrm{e}^{\mathrm{i} p x} Q_{J, E}(2 k x)$ of (2.1). In view of Proposition 2.7 we can use $T$ and $\Psi$ (instead of $J$ and $E$ ) to parametrize the family of quasi-periodic solutions of (2.1). For our present purposes, the most convenient set of parameters will be $(k, p)$, where $p=k+\ell$ and $k, \ell$ are defined in (2.27). 
Since the equation (2.1) does not depend upon $k$ and $p$, the derivatives $\partial W_{J, E} / \partial k$ and $\partial W_{J, E} / \partial p$ are solutions of the linear equation

$$
\omega^{\prime \prime}+\omega-2\left|W_{J, E}\right|^{2} \omega-W_{J, E}^{2} \bar{\omega}=0 .
$$

It is then straightforward to check that the functions $R_{1}(z)$ and $R_{2}(z)$ defined for $z=2 k x$ through

$$
\begin{aligned}
& R_{1}(2 k x)=\mathrm{e}^{-\mathrm{i} p x} \frac{\partial W_{J, E}}{\partial k}(x)=\frac{\partial Q_{J, E}}{\partial k}(2 k x)+2 x Q_{J, E}^{\prime}(2 k x), \\
& R_{2}(2 k x)=\mathrm{e}^{-\mathrm{i} p x} \frac{\partial W_{J, E}}{\partial p}(x)=\frac{\partial Q_{J, E}}{\partial p}(2 k x)+\mathrm{i} x Q_{J, E}(2 k x),
\end{aligned}
$$

satisfy $H_{J, E} R_{1}=H_{J, E} R_{2}=0$, namely $R_{1}, R_{2} \in K$. Of course $R_{1}, R_{2}$ depend on the point $(J, E) \in D$ where the derivative is taken, but we omit this dependence for notational simplicity.

Now, let $R(z)=A_{1} R_{1}(z)+A_{2} R_{2}(z)$ for some $A_{1}, A_{2} \in \mathbb{R}$. Using the definitions (3.7) and the fact that $Q_{J, E}$ is $2 \pi$-periodic we find that $R$ satisfies the periodicity conditions $R(2 \pi)=R(0)$ and $R^{\prime}(2 \pi)=R^{\prime}(0)$ if and only if

$$
2 A_{1} Q_{J, E}^{\prime}(0)+\mathrm{i} A_{2} Q_{J, E}(0)=0, \quad \text { and } \quad 2 A_{1} Q_{J, E}^{\prime \prime}(0)+\mathrm{i} A_{2} Q_{J, E}^{\prime}(0)=0 .
$$

This linear system has a nontrivial solution $\left(A_{1}, A_{2}\right) \in \mathbb{C}^{2}$ if and only if

$$
Q_{J, E}^{\prime}(0)^{2}=Q_{J, E}(0) Q_{J, E}^{\prime \prime}(0)
$$

Using again the quasi-periodic solution $W_{J, E}(x)$ which satisfies the initial conditions $W_{J, E}(0)=r_{2}$, $W_{J, E}^{\prime}(0)=\mathrm{i} J / r_{2}$ and $W_{J, E}^{\prime \prime}(0)=r_{2}^{3}-r_{2}$, it is not difficult to verify that (3.8) is equivalent to $J^{2}=r_{2}^{4}-r_{2}^{6}$. But we know that $J^{2}=r_{Q}^{4}-r_{Q}^{6}=r_{q}^{4}-r_{q}^{6}$ with $r_{Q}<r_{2}<r_{q}$ (see Fig. 2), hence $J^{2}<r_{2}^{4}-r_{2}^{6}$ so that (3.8) never holds. We conclude that $R=A_{1} R_{1}+A_{2} R_{2}$ belongs to $X_{2}$ only if $A_{1}=A_{2}=0$. This shows that the four functions $Q_{J, E}^{\prime}, \mathrm{i} Q_{J, E}, R_{1}, R_{2}$ are linearly independent $\left(\right.$ over $\mathbb{R}$ ) and therefore form a basis of the kernel $K$. Moreover, $K \cap X_{2}=\operatorname{span}\left\{Q_{J, E}^{\prime}, \mathrm{i} Q_{J, E}\right\}$ as expected.

It is now easy to conclude the proof of Proposition 3.2 Indeed, since the eigenvalues of $H_{J, E}$ depend continuously on $(J, E) \in D$, the fact that the zero eigenvalue has constant multiplicity implies that the number of negative eigenvalues of $H_{J, E}$ remains unchanged when $(J, E)$ varies over $D$. It is therefore sufficient to verify that the conclusion of Proposition 3.2 holds for one value of $(J, E) \in D$. But as $(J, E) \rightarrow 0$ the wave profile $Q_{J, E}$ converges uniformly to zero, hence $H_{J, E}$ converges to a constant coefficient operator whose spectrum is easily determined by Fourier analysis. Using a perturbation argument, it is then straightforward to localize the eigenvalues of $H_{J, E}$ for $(J, E)$ close to the origin. This calculation is performed in [12], using an appropriate parametrization of the small amplitude periodic waves. In the particular case $J=0$, we obtain the result below, which concludes the proof of Proposition 3.2 .

Lemma 3.3 ([12, Proposition A.1]) If $J=0$ and $E>0$ is sufficiently small, the operator $H_{J, E}$ acting on $X_{0}$ has exactly three eigenvalues $\left\{\lambda_{1}, \lambda_{2}, 0\right\}$ in a neighborhood of the origin, and the rest 
of its spectrum is positive and bounded away from zero. Moreover, the eigenvalues $\lambda_{1}, \lambda_{2}$ are simple and satisfy ${ }^{1}$

$$
\lambda_{1}(E)=-E+\mathcal{O}\left(E^{2}\right), \quad \lambda_{2}(E)=3 E+\mathcal{O}\left(E^{2}\right), \quad \text { as } E \rightarrow 0 .
$$

Remark 3.4 By construction the wave profile $W_{J, E}(x)=\mathrm{e}^{\mathrm{i} p x} Q_{J, E}(2 k x)$ is a stationary solution of the time-dependent Ginzburg-Landau equation

$$
W_{t}(x, t)=W_{x x}(x, t)+W(x, t)-|W(x, t)|^{2} W(x, t) .
$$

If we look for solutions of (3.9) of the form $W(x, t)=\mathrm{e}^{\mathrm{i} p x} Q(2 k x, t)$, we obtain the evolution equation

$$
Q_{t}(z, t)=4 k^{2} Q_{z z}(z, t)+4 \mathrm{i} p k Q_{z}(z, t)+\left(1-p^{2}\right) Q(z, t)-|Q(z, t)|^{2} Q(z, t),
$$

which is very similar to (3.2). In particular, the linearization of (3.10) at the equilibrium $Q_{J, E}$ is $Q_{t}=-H_{J, E} Q$. Thus Proposition 3.2 implies that the quasi-periodic solutions of the GinzburgLandau equation (3.9) are always unstable, even within the class of solutions with the same period and the same Floquet multiplier as the original wave profile. This complements previous results by Bridges 8 Rowlands [5], and by Doelman, Gardner 8 Jones [9], which show that the quasi-periodic solutions of (3.9) are unstable with respect to long-wave disturbances ("sideband instability").

We now continue with the proof of Proposition 3.1. The next observation is that, for any $(J, E) \in D$, the equilibrium $Q_{J, E}$ of (3.2) is a member of a two-parameter family of travelling and rotating waves of the form

$$
Q(z, t)=\mathrm{e}^{-\mathrm{i} \omega t} Q_{J, E}^{\omega, c}(z+c t), \quad z \in \mathbb{R}, \quad t \in \mathbb{R},
$$

where $(\omega, c)$ lies in a neighborhood of the origin in $\mathbb{R}^{2}$ (the Lie algebra of $G$ ), and the profile $Q_{J, E}^{\omega, c} \in X$ is a smooth function of $(\omega, c)$ with $Q_{J, E}^{0,0}=Q_{J, E}$. (Actually we even have a four-parameter family of such waves if we take into account the action of the symmetry group $G$.) Indeed, take $\left(J^{\prime}, E^{\prime}\right) \in D$ close to $(J, E)$, and define $k^{\prime}, \ell^{\prime}$ by the formulas (2.27) with $(J, E)$ replaced by $\left(J^{\prime}, E^{\prime}\right)$. Then

$$
U(x, t)=\mathrm{e}^{\mathrm{i}\left(p^{\prime} x-t\right)} Q_{J^{\prime}, E^{\prime}}\left(2 k^{\prime} x\right), \quad \text { where } p^{\prime}=k^{\prime}+\ell^{\prime},
$$

is a solution of (1.1), but it is not of the form (3.1) because $p^{\prime} \neq p$ and $k^{\prime} \neq k$ in general. However we can transform $U(x, t)$ into a solution of (1.1) of the form (3.1), (3.11) by applying successively a dilation of factor $\lambda$ and a Galilean transformation of speed $v$, where

$$
\lambda=\frac{k}{k^{\prime}}, \quad v=2\left(\lambda p^{\prime}-p\right) .
$$

After some elementary algebra, we obtain $Q_{J, E}^{\omega, c}(z)=\lambda Q_{J^{\prime}, E^{\prime}}(z)$ with

$$
\omega=\lambda^{2}\left(1-p^{2}\right)-\left(1-p^{2}\right), \quad c=4 \lambda^{2} k^{\prime} p^{\prime}-4 k p .
$$

\footnotetext{
${ }^{1}$ The expansions of $\lambda_{1}, \lambda_{2}$ do not appear explicitly in 12, but they follow easily from the proof of Proposition A.1 there if one observes that the case $J=0$ corresponds to $a=b$ and $E=2 a^{2}+\mathcal{O}\left(a^{4}\right)$ in the notation of [12].
} 
The mapping $\left(J^{\prime}, E^{\prime}\right) \rightarrow(\omega, c)$ that we have just defined is a diffeomorphism from a neighborhood of $(J, E)$ onto a neighborhood of $(0,0)$. Indeed, using (2.27) and (3.12), the formulas (3.13) can be written more explicitly as

$$
\omega=\frac{k^{2}}{\pi^{2}}\left(T^{\prime 2}-\left(\pi+\Psi^{\prime}\right)^{2}\right)-\left(1-p^{2}\right), \quad c=\frac{4 k^{2}}{\pi}\left(\pi+\Psi^{\prime}\right)-4 k p,
$$

where $T^{\prime}=T\left(J^{\prime}, E^{\prime}\right)$ and $\Psi^{\prime}=\Psi\left(J^{\prime}, E^{\prime}\right)$. Differentiating these expressions with respect to $J^{\prime}, E^{\prime}$ we obtain

$$
\left.\mathcal{M}_{J, E} \stackrel{\text { def }}{=}\left(\begin{array}{ll}
\frac{\partial \omega}{\partial E^{\prime}} & \frac{\partial c}{\partial E^{\prime}} \\
\frac{\partial \omega}{\partial J^{\prime}} & \frac{\partial c}{\partial J^{\prime}}
\end{array}\right)\right|_{\left(J^{\prime}, E^{\prime}\right)=(J, E)}=\left(\begin{array}{ll}
\frac{2 k}{\pi}\left(\frac{\partial T}{\partial E}-p \frac{\partial \Psi}{\partial E}\right) & \frac{4 k^{2}}{\pi} \frac{\partial \Psi}{\partial E} \\
\frac{2 k}{\pi}\left(\frac{\partial T}{\partial J}-p \frac{\partial \Psi}{\partial J}\right) & \frac{4 k^{2}}{\pi} \frac{\partial \Psi}{\partial J}
\end{array}\right) .
$$

By Proposition 2.6] we have $\operatorname{det}\left(\mathcal{M}_{J, E}\right)=\left(8 k^{3} / \pi^{2}\right) \Delta(J, E)>0$, hence the matrix $\mathcal{M}_{J, E}$ is invertible. This proves the existence of the travelling and rotating waves (3.11) for sufficiently small $(\omega, c) \in \mathbb{R}^{2}$.

By construction, the profile $Q_{J, E}^{\omega, c} \in X$ is a critical point of the functional

$$
\mathcal{E}_{J, E}^{\omega, c}(Q)=\mathcal{E}_{J, E}(Q)-\omega N(Q)-c M(Q), \quad Q \in X
$$

For later use, we remark that, if $\lambda$ is as in (3.12), we have the identity

$$
\mathcal{E}_{J, E}^{\omega, c}(\lambda Q)=\lambda^{4} \mathcal{E}_{J^{\prime}, E^{\prime}}(Q), \quad \text { for all } Q \in X \text {. }
$$

Following [16] we now define, for $(\omega, c)$ in a neighborhood of $(0,0)$,

$$
d_{J, E}(\omega, c)=\mathcal{E}_{J, E}^{\omega, c}\left(Q_{J, E}^{\omega, c}\right) \equiv \lambda^{4} \mathcal{E}_{J^{\prime}, E^{\prime}}\left(Q_{J^{\prime}, E^{\prime}}\right)
$$

A crucial role in the orbital stability argument will be played by the Hessian matrix of $d_{J, E}$, namely:

$$
\mathcal{H}_{J, E}=\left.\left(\begin{array}{ll}
\frac{\partial^{2} d_{J, E}}{\partial \omega^{2}} & \frac{\partial^{2} d_{J, E}}{\partial \omega \partial c} \\
\frac{\partial^{2} d_{J, E}}{\partial c \partial \omega} & \frac{\partial^{2} d_{J, E}}{\partial c^{2}}
\end{array}\right)\right|_{(\omega, c)=(0,0)} .
$$

Proposition 3.5 For all $(J, E) \in D$ we have $\operatorname{det}\left(\mathcal{H}_{J, E}\right)<0$.

Remark 3.6 Since $\mathcal{H}_{J, E}$ is a symmetric matrix, Proposition 3.5 implies of course that $\mathcal{H}_{J, E}$ has one positive and one negative eigenvalue. This can be verified explicitly in the case of small amplitude periodic waves, for which the following asymptotic result is established in [12]:

$$
\mathcal{H}_{J, E}=\frac{\pi}{3}\left(\begin{array}{cc}
-2 & -1 \\
-1 & 1
\end{array}\right)(\mathbf{1}+\mathcal{O}(E)), \quad \text { as } \quad(J, E) \rightarrow(0,0) \text { in } D .
$$

Since the proof of Proposition 3.5 is long and technical, we postpone it to the end of this section, and we now show how Propositions 3.2 and 3.5 together imply Proposition 3.1. The arguments here are rather classical and can be found in [16], so we shall just indicate how the general theory of [16] applies to present case.

For any $(J, E) \in D$, Proposition 3.2 shows that the wave profile $Q_{J, E}$ is a degenerate saddle point of the energy $\mathcal{E}_{J, E}$, with one unstable and two neutral directions (the latter are due to the 
fact that (3.2) is $G$-invariant). To get rid of the unstable direction we remark that the evolution of (3.2) does not take place in the whole function space $X$, but on codimension two surfaces where the charge $N$ and the momentum $M$ are constant. Let

$$
\Sigma_{J, E}=\left\{Q \in X \mid N(Q)=N\left(Q_{J, E}\right), M(Q)=M\left(Q_{J, E}\right)\right\} .
$$

It is easy to verify that $\Sigma_{J, E}$ is indeed a smooth submanifold of $X$ of codimension two in a neighborhood of $Q_{J, E}$, or more generally in a neighborhood of the orbit of $Q_{J, E}$ under $G$. The crucial point is that the functional $\mathcal{E}_{J, E}$ is coercive on $\Sigma_{J, E}$ with respect to the semi-distance $\rho$ defined by (3.3). More precisely, there exist positive constants $C_{1}, \delta$ (depending on $J, E$ ) such that

$$
\mathcal{E}_{J, E}(Q)-\mathcal{E}_{J, E}\left(Q_{J, E}\right) \geq C_{1} \rho\left(Q, Q_{J, E}\right)^{2},
$$

for all $Q \in \Sigma_{J, E}$ such that $\rho\left(Q, Q_{J, E}\right) \leq \delta$.

To prove (3.20) we first note that the tangent space to $\Sigma_{J, E}$ at $Q_{J, E}$ is

$$
\mathcal{T}_{J, E}=\left\{Q \in X \mid\left\langle Q_{J, E}, Q\right\rangle=\left\langle\mathrm{i} Q_{J, E}^{\prime}, Q\right\rangle=0\right\},
$$

(see (3.5)). We next introduce the "normal space" $\mathcal{N}_{J, E}=\operatorname{span}\left\{\partial_{\omega} Q_{J, E}, \partial_{c} Q_{J, E}\right\}$, where

$$
\partial_{\omega} Q_{J, E}=\left.\frac{\partial}{\partial \omega} Q_{J, E}^{\omega, c}\right|_{(\omega, c)=(0,0)}, \quad \partial_{c} Q_{J, E}=\left.\frac{\partial}{\partial c} Q_{J, E}^{\omega, c}\right|_{(\omega, c)=(0,0)} .
$$

As $Q_{J, E}^{\omega, c}$ is a critical point of $\mathcal{E}_{J, E}^{\omega, c}$, we have $\mathcal{E}_{J, E}^{\prime}\left(Q_{J, E}^{\omega, c}\right)=\omega N^{\prime}\left(Q_{J, E}^{\omega, c}\right)+c M^{\prime}\left(Q_{J, E}^{\omega, c}\right)$. Differentiating this relation with respect to $\omega$ and $c$ at $(\omega, c)=(0,0)$, we obtain

$$
H_{J, E}\left(\partial_{\omega} Q_{J, E}\right)=N^{\prime}\left(Q_{J, E}\right)=Q_{J, E}, \quad H_{J, E}\left(\partial_{c} Q_{J, E}\right)=M^{\prime}\left(Q_{J, E}\right)=\mathrm{i} Q_{J, E}^{\prime},
$$

hence $\left\langle H_{J, E} Q_{1}, Q_{2}\right\rangle=0$ for all $Q_{1} \in \mathcal{N}_{J, E}, Q_{2} \in \mathcal{T}_{J, E}$. In a similar way, differentiating (3.18) we find

$$
\frac{\partial}{\partial \omega} d_{J, E}(\omega, c)=-N\left(Q_{J, E}^{\omega, c}\right), \quad \frac{\partial}{\partial c} d_{J, E}(\omega, c)=-M\left(Q_{J, E}^{\omega, c}\right),
$$

hence

$$
\begin{aligned}
\mathcal{H}_{J, E} & =-\left.\left(\begin{array}{cc}
\frac{\partial}{\partial \omega} N\left(Q_{J, E}^{\omega, c}\right) & \frac{\partial}{\partial \omega} M\left(Q_{J, E}^{\omega, c}\right) \\
\frac{\partial}{\partial c} N\left(Q_{J, E}^{\omega, c}\right) & \frac{\partial}{\partial c} M\left(Q_{J, E}^{\omega, c}\right)
\end{array}\right)\right|_{(\omega, c)=(0,0)} \\
& =-\left(\begin{array}{cc}
\left\langle H_{J, E}\left(\partial_{\omega} Q_{J, E}\right), \partial_{\omega} Q_{J, E}\right\rangle & \left\langle H_{J, E}\left(\partial_{c} Q_{J, E}\right), \partial_{\omega} Q_{J, E}\right\rangle \\
\left\langle H_{J, E}\left(\partial_{\omega} Q_{J, E}\right), \partial_{c} Q_{J, E}\right\rangle & \left\langle H_{J, E}\left(\partial_{c} Q_{J, E}\right), \partial_{c} Q_{J, E}\right\rangle
\end{array}\right) .
\end{aligned}
$$

Thus $-\mathcal{H}_{J, E}$ is the matrix of the bilinear form $b_{J, E}\left(Q_{1}, Q_{2}\right)=\left\langle H_{J, E} Q_{1}, Q_{2}\right\rangle$ restricted to the subspace $\mathcal{N}_{J, E}$. As $\mathcal{H}_{J, E}$ is non-degenerate by Proposition 3.5. we have $\mathcal{N}_{J, E} \cap \mathcal{T}_{J, E}=\{0\}$, hence also $X=\mathcal{N}_{J, E} \oplus \mathcal{T}_{J, E}$. Moreover, since $H_{J, E}$ has only one negative eigenvalue by Proposition 3.2 and since the restriction of $b_{J, E}$ to $\mathcal{N}_{J, E}$ has a negative eigenvalue by Proposition [3.5, a standard signature argument shows that the restriction of $b_{J, E}$ to the complementary space $\mathcal{T}_{J, E}$ is nonnegative. More precisely, if we decompose $\mathcal{T}_{J, E}=K_{J, E} \oplus \mathcal{T}_{J, E}^{\prime}$ where $K_{J, E}=\operatorname{span}\left\{Q_{J, E}^{\prime}, \mathrm{i} Q_{J, E}\right\}$ is the kernel of $H_{J, E}$ in $X$ and $\mathcal{T}_{J, E}^{\prime}=\left\{Q \in \mathcal{T}_{J, E} \mid\left\langle Q_{J, E}^{\prime}, Q\right\rangle=\left\langle\mathrm{i} Q_{J, E}, Q\right\rangle=0\right\}$, then there exists $C_{2}>0$ such that

$$
\left\langle H_{J, E} Q, Q\right\rangle \geq C_{2}\|Q\|_{X}^{2}, \quad \text { for all } Q \in \mathcal{T}_{J, E}^{\prime} \text {. }
$$

Estimate (3.20) follows from its infinitesimal version (3.24) using a Taylor expansion of the energy $\mathcal{E}_{J, E}$ at $Q_{J, E}$ and the fact that $\mathcal{E}_{J, E}$ is $G$-invariant (see [16] for details). 
Remark 3.7 The argument above does not give any lower bound on the constants $C_{1}, C_{2}$ in (3.20), 3.24). In particular, we cannot take for $C_{2}$ the lowest positive eigenvalue of the operator $H_{J, E}$, unless the subspace $\mathcal{N}_{J, E}$ contains precisely the eigenfunction associated to the (unique) negative eigenvalue of $H_{J, E}$. However, one can check that the constants $C_{1}, \delta$ in (3.20) are bounded away from zero uniformly for $(J, E)$ in any compact subdomain of $D$.

With inequality (3.20) at hand, it is now easy to conclude the proof of Proposition 3.1] Assume that $Q_{0} \in X$ satisfies $\rho\left(Q_{0}, Q_{J, E}\right) \leq \epsilon$ for some small $\epsilon>0$. Replacing $Q_{0}$ by $\mathcal{R}_{(\varphi, \xi)} Q_{0}$ if needed, we can assume that $\left\|Q_{0}-Q_{J, E}\right\|_{X} \leq \epsilon$. As $Q_{J, E}$ is a critical point of $\mathcal{E}_{J, E}$, we have $\mid \mathcal{E}_{J, E}\left(Q_{0}\right)-$ $\mathcal{E}_{J, E}\left(Q_{J, E}\right) \mid \leq C_{3} \epsilon^{2}$, for some $C_{3}>0$ (depending on $J, E$ ). We distinguish two cases:

1. If $Q_{0} \in \Sigma_{J, E}$, then the solution of (3.2) with initial data $Q_{0}$ satisfies $Q(\cdot, t) \in \Sigma_{J, E}$ for all $t \in \mathbb{R}$, and (3.20) implies that

$$
C_{1} \rho\left(Q(\cdot, t), Q_{J, E}\right)^{2} \leq \mathcal{E}_{J, E}(Q(\cdot, t))-\mathcal{E}_{J, E}\left(Q_{J, E}\right)=\mathcal{E}_{J, E}\left(Q_{0}\right)-\mathcal{E}_{J, E}\left(Q_{J, E}\right) \leq C_{3} \epsilon^{2},
$$

for all $t \in \mathbb{R}$, provided $\epsilon$ is small enough so that $C_{3} \epsilon^{2}<C_{1} \delta^{2}$. This is the desired result.

2. The case where $Q_{0} \notin \Sigma_{J, E}$ can be reduced to the previous one by the following argument. In view of (3.22) and Proposition 3.5, the map $(\omega, c) \mapsto\left(N\left(Q_{J, E}^{\omega, c}\right), M\left(Q_{J, E}^{\omega, c}\right)\right)$ is a diffeomorphism from a neighborhood of $(0,0)$ onto a neighborhood of $\left(N\left(Q_{J, E}\right), M\left(Q_{J, E}\right)\right)$. Thus there exists a unique $(\omega, c) \in \mathbb{R}^{2}$ with $|\omega|+|c|=\mathcal{O}(\epsilon)$ such that $N\left(Q_{0}\right)=N\left(Q_{J, E}^{\omega, c}\right), M\left(Q_{0}\right)=M\left(Q_{J, E}^{\omega, c}\right)$. By construction $Q_{J, E}^{\omega, c}=\lambda Q_{J^{\prime}, E^{\prime}}$, where $\left(J^{\prime}, E^{\prime}\right)$ is $\epsilon$-close to $(J, E)$ and $\lambda$ is as in (3.12). Thus, if we denote $\tilde{Q}(z, t)=\lambda^{-1} Q(z, t)$, we see that $\tilde{Q}(\cdot, t) \in \Sigma_{J^{\prime}, E^{\prime}}$ for all $t \in \mathbb{R}$. Using (3.17) together with (3.20), we thus obtain

$$
\begin{aligned}
\mathcal{E}_{J, E}^{\omega, c}(Q(\cdot, t))-\mathcal{E}_{J, E}^{\omega, c}\left(Q_{J, E}^{\omega, c}\right) & =\lambda^{4}\left(\mathcal{E}_{J^{\prime}, E^{\prime}}(\tilde{Q}(\cdot, t))-\mathcal{E}_{J^{\prime}, E^{\prime}}\left(Q_{J^{\prime}, E^{\prime}}\right)\right) \\
& \geq C_{1}^{\prime} \lambda^{4} \rho\left(\tilde{Q}(\cdot, t), Q_{J^{\prime}, E^{\prime}}\right)^{2}=C_{1}^{\prime} \lambda^{2} \rho\left(Q(\cdot, t), Q_{J, E}^{\omega, c}\right)^{2}
\end{aligned}
$$

as long as $\rho\left(Q(\cdot, t), Q_{J, E}^{\omega, c}\right)$ stays sufficiently small. Since

$$
\mathcal{E}_{J, E}^{\omega, c}(Q(\cdot, t))-\mathcal{E}_{J, E}^{\omega, c}\left(Q_{J, E}^{\omega, c}\right)=\mathcal{E}_{J, E}^{\omega, c}\left(Q_{0}\right)-\mathcal{E}_{J, E}^{\omega, c}\left(Q_{J, E}^{\omega, c}\right) \leq C_{3}^{\prime}\left\|Q_{0}-Q_{J, E}^{\omega, c}\right\|_{X}^{2},
$$

and $\left\|Q_{0}-Q_{J, E}^{\omega, c}\right\|_{X} \leq\left\|Q_{0}-Q_{J, E}\right\|_{X}+\left\|Q_{J, E}-Q_{J, E}^{\omega, c}\right\|_{X} \leq C_{4} \epsilon$, we conclude that

$$
\rho\left(Q(\cdot, t), Q_{J, E}\right) \leq \rho\left(Q(\cdot, t), Q_{J, E}^{\omega, c}\right)+\left\|Q_{J, E}^{\omega, c}-Q_{J, E}\right\|_{X} \leq C_{5} \epsilon \quad \text { for all } t \in \mathbb{R},
$$

if $\epsilon>0$ is sufficiently small. This concludes the proof of Proposition 3.1.

Proof of Proposition 3.5. We start from the expression (3.22) of $\mathcal{H}_{J, E}$. We recall that $(\omega, c)$ can be parametrized (in a neighborhood of the origin) by $\left(J^{\prime}, E^{\prime}\right)$ according to the formulas (3.13) or (3.14), and that $Q_{J, E}^{\omega, c}=\lambda Q_{J^{\prime}, E^{\prime}}$ where $\lambda$ is given by (3.12). Thus $\mathcal{H}_{J, E}=-\left(\mathcal{M}_{J, E}\right)^{-1} \mathcal{K}_{J, E}$, where $\mathcal{M}_{J, E}$ is defined in (3.15) and

$$
\mathcal{K}_{J, E}=\left.\left(\begin{array}{ll}
\frac{\partial^{\prime} N}{\partial^{\prime} E} & \frac{\partial^{\prime} M}{\partial^{\prime} E} \\
\frac{\partial^{\prime} N}{\partial^{\prime} J} & \frac{\partial^{\prime} M}{\partial^{\prime} J}
\end{array}\right) \stackrel{\text { def }}{=}\left(\begin{array}{cc}
\frac{\partial}{\partial E^{\prime}} N\left(\lambda Q_{J^{\prime}, E^{\prime}}\right) & \frac{\partial}{\partial E^{\prime}} M\left(\lambda Q_{J^{\prime}, E^{\prime}}\right) \\
\frac{\partial}{\partial J^{\prime}} N\left(\lambda Q_{J^{\prime}, E^{\prime}}\right) & \frac{\partial}{\partial J^{\prime}} M\left(\lambda Q_{J^{\prime}, E^{\prime}}\right)
\end{array}\right)\right|_{\left(J^{\prime}, E^{\prime}\right)=(J, E)} .
$$

Since we already know that $\operatorname{det}\left(\mathcal{M}_{J, E}\right)>0$, it remains to verify that $\operatorname{det}\left(\mathcal{K}_{J, E}\right)<0$. 
We first give more explicit formulas for the coefficients of $\mathcal{K}_{J, E}$. As $W_{J, E}(x)=\mathrm{e}^{\mathrm{i} p x} Q_{J, E}(2 k x)$, where $p=k+\ell$ and $k, \ell$ are given by (2.27), it follows from (3.4) that

$$
\begin{aligned}
N\left(Q_{J, E}\right) & =\frac{1}{2} \int_{0}^{2 \pi}\left|Q_{J, E}(z)\right|^{2} \mathrm{~d} z=k \int_{0}^{T}\left|W_{J, E}(x)\right|^{2} \mathrm{~d} x, \\
M\left(Q_{J, E}\right) & =\frac{\mathrm{i}}{2} \int_{0}^{2 \pi} \bar{Q}_{J, E}(z) Q_{J, E}^{\prime}(z) \mathrm{d} z \\
& =\frac{\mathrm{i}}{2} \int_{0}^{T}\left(\bar{W}_{J, E}(x) W_{J, E}^{\prime}(x)-\mathrm{i} p\left|W_{J, E}(x)\right|^{2}\right) \mathrm{d} x=\frac{p}{2 k} N\left(Q_{J, E}\right)-\frac{1}{2} J T .
\end{aligned}
$$

Moreover, proceeding as in Lemma 2.2 we find

$$
N\left(Q_{J, E}\right)=2 k \int_{r_{1}(J, E)}^{r_{2}(J, E)} \frac{r^{2}}{\sqrt{2\left(E-V_{J}(r)\right)}} \mathrm{d} r=2 \sqrt{2} k \int_{0}^{\pi / 2} \frac{s(\varphi)}{\sqrt{y_{3}-s(\varphi)}} \mathrm{d} \varphi,
$$

where $s(\varphi)=y_{1} \cos ^{2}(\varphi)+y_{2} \sin ^{2}(\varphi)$ and $y_{1}, y_{2}, y_{3}$ are as in Fig. 3. Of course, since $N, M$ are quadratic functionals, we have

$$
N\left(\lambda Q_{J^{\prime}, E^{\prime}}\right)=\lambda^{2} N\left(Q_{J^{\prime}, E^{\prime}}\right)=\frac{k^{2}}{k^{2}} N\left(Q_{J^{\prime}, E^{\prime}}\right), \quad M\left(\lambda Q_{J^{\prime}, E^{\prime}}\right)=\frac{k^{2}}{k^{2}} M\left(Q_{J^{\prime}, E^{\prime}}\right) .
$$

Differentiating (3.27) with respect to $J^{\prime}, E^{\prime}$ and using (3.25), (3.26), we obtain after straightforward calculations:

$$
\begin{aligned}
& \frac{\partial^{\prime} N}{\partial^{\prime} E}=C_{1} \frac{\partial y_{1}}{\partial E}+C_{2} \frac{\partial y_{2}}{\partial E}+C_{3} \frac{\partial T}{\partial E}, \quad \frac{\partial^{\prime} N}{\partial^{\prime} J}=C_{1} \frac{\partial y_{1}}{\partial J}+C_{2} \frac{\partial y_{2}}{\partial J}+C_{3} \frac{\partial T}{\partial J} \\
& \frac{\partial^{\prime} M}{\partial^{\prime} E}=\frac{p}{2 k} \frac{\partial^{\prime} N}{\partial^{\prime} E}+\frac{N}{2 \pi} \frac{\partial \Psi}{\partial E}-\frac{3 J}{2} \frac{\partial T}{\partial E}, \quad \frac{\partial^{\prime} M}{\partial^{\prime} J}=\frac{p}{2 k} \frac{\partial^{\prime} N}{\partial^{\prime} J}+\frac{N}{2 \pi} \frac{\partial \Psi}{\partial J}-\frac{3 J}{2} \frac{\partial T}{\partial J}-\frac{T}{2},
\end{aligned}
$$

where $N=N\left(Q_{J, E}\right)$ and

$$
\begin{aligned}
C_{1} & =\sqrt{2} k \int_{0}^{\pi / 2} \frac{2 y_{3} \cos ^{2}(\varphi)+s(\varphi) \sin ^{2}(\varphi)}{\left(y_{3}-s(\varphi)\right)^{3 / 2}} \mathrm{~d} \varphi, \\
C_{2} & =\sqrt{2} k \int_{0}^{\pi / 2} \frac{2 y_{3} \sin ^{2}(\varphi)+s(\varphi) \cos ^{2}(\varphi)}{\left(y_{3}-s(\varphi)\right)^{3 / 2}} \mathrm{~d} \varphi, \\
C_{3} & =2 \sqrt{2} \frac{k^{2}}{\pi} \int_{0}^{\pi / 2} \frac{s(\varphi)}{\sqrt{y_{3}-s(\varphi)}} \mathrm{d} \varphi=\frac{k}{\pi} N\left(Q_{J, E}\right) .
\end{aligned}
$$

We now compute $\operatorname{det}\left(\mathcal{K}_{J, E}\right)$. Replacing the second column of the matrix $\mathcal{K}_{J, E}$ by its expression (3.29), we see that $\operatorname{det}\left(\mathcal{K}_{J, E}\right)=\Delta_{1}+\Delta_{2}+\Delta_{3}$, where

$$
\Delta_{1}=\left(\frac{N B_{3}}{2 \pi}-\frac{T}{2}\right) \frac{\partial^{\prime} N}{\partial^{\prime} E}
$$

and

$$
\Delta_{2}=\frac{N}{2 \pi} \operatorname{det}\left(\begin{array}{cc}
\frac{\partial^{\prime} N}{\partial^{\prime} E} & \frac{\partial \Psi}{\partial E} \\
\frac{\partial^{\prime} N}{\partial^{\prime} J} & \frac{\partial \Psi}{\partial J}-B_{3}
\end{array}\right), \quad \Delta_{3}=-\frac{3 J}{2} \operatorname{det}\left(\begin{array}{cc}
\frac{\partial^{\prime} N}{\partial^{\prime} E} & \frac{\partial T}{\partial E} \\
\frac{\partial^{\prime} N}{\partial^{\prime} J} & \frac{\partial T}{\partial J}
\end{array}\right) .
$$

Here $B_{3}$ is as in (2.19). 
We claim that $\Delta_{1}<0$. Indeed, arguing as in (2.15) and using Lemma 2.5, we find

$$
C_{2}-C_{1}=\sqrt{2} k \int_{0}^{\pi / 2} \frac{2 y_{3}-s(\varphi)}{\left(y_{3}-s(\varphi)\right)^{3 / 2}}\left(\sin ^{2}(\varphi)-\cos ^{2}(\varphi)\right) \mathrm{d} \varphi>0
$$

because $\varphi \mapsto\left(2 y_{3}-s(\varphi)\right)\left(y_{3}-s(\varphi)\right)^{-3 / 2}$ is strictly increasing over $[0, \pi / 2]$. Thus $C_{2}>C_{1}>0$, and proceeding as in (2.16) we deduce that $C_{1}\left(\partial y_{1} / \partial E\right)+C_{2}\left(\partial y_{2} / \partial E\right)>0$. Since $C_{3}>0$ and $\partial T / \partial E>0$ by Proposition 2.4 we conclude that $\partial^{\prime} N / \partial^{\prime} E>0$. On the other hand, since $s(\varphi) \leq y_{2}$ for all $\varphi \in[0,2 \pi]$, it follows from (2.7), (2.27), (3.26) that $N \leq \pi y_{2}$. Moreover, as

$$
\frac{y_{2}}{\sqrt{y_{3}}\left(\sqrt{y_{3}}+\sqrt{y_{3}-s(\varphi)}\right)} \leq \frac{y_{2}}{\sqrt{y_{3}}\left(\sqrt{y_{3}}+\sqrt{y_{3}-y_{2}}\right)}=\frac{\sqrt{y_{3}}-\sqrt{y_{3}-y_{2}}}{\sqrt{y_{3}}}<1,
$$

it follows from (2.7), (2.20) that $B_{3} y_{2}<T$. Thus $N B_{3}<\pi T$, hence $\Delta_{1}<0$.

It remains to verify that $\Delta_{2}+\Delta_{3}<0$. By (2.14), (3.28) we have

$$
\frac{\partial^{\prime} N}{\partial^{\prime} E}=\left(C_{1}+C_{3} A_{1}\right) \frac{\partial y_{1}}{\partial E}+\left(C_{2}+C_{3} A_{2}\right) \frac{\partial y_{2}}{\partial E},
$$

and similarly for the $J$-derivative. Thus using (2.19) and proceeding as in (2.21) we find

$$
\Delta_{2}=\frac{N}{2 \pi}\left(y_{2}-y_{1}\right) \frac{\partial y_{1}}{\partial J} \frac{\partial y_{2}}{\partial J}\left(\left(C_{1}+C_{3} A_{1}\right) B_{2}-\left(C_{2}+C_{3} A_{2}\right) B_{1}\right) .
$$

In a similar way

$$
\Delta_{3}=-\frac{3}{2}\left(y_{2}-y_{1}\right) \frac{\partial y_{1}}{\partial J} \frac{\partial y_{2}}{\partial J}\left(C_{1} A_{2}-C_{2} A_{1}\right) .
$$

Thus $\Delta_{2}+\Delta_{3}=\left(y_{2}-y_{1}\right)\left(\partial y_{1} / \partial J\right)\left(\partial y_{2} / \partial J\right) \Delta_{4}$, where

$$
\Delta_{4}=\frac{3}{2}\left(A_{1} C_{2}-A_{2} C_{1}\right)+\frac{N C_{3}}{2 \pi}\left(A_{1} B_{2}-A_{2} B_{1}\right)+\frac{N}{2 \pi}\left(C_{1} B_{2}-C_{2} B_{1}\right) .
$$

As $y_{2}>y_{1}$ and $\left(\partial y_{1} / \partial J\right)\left(\partial y_{2} / \partial J\right)<0$, we have to verify that $\Delta_{4}>0$.

Like in the proof of Proposition [2.6] it is convenient to express the various constants $A_{i}, B_{i}, C_{i}$ in terms of the new function $\sigma(\varphi)=\left(1-y_{3}^{-1} s(\varphi)\right)^{1 / 2}$. Then $A_{i}=\sqrt{2} y_{3}^{-3 / 2} \mathbf{A}_{i}, B_{i}=\sqrt{2} y_{3}^{-5 / 2} \mathbf{B}_{i}$, $C_{i}=\sqrt{2} k y_{3}^{-1 / 2} \mathbf{C}_{i}$, where $\mathbf{A}_{i}, \mathbf{B}_{i}$ are defined in (2.22) and

$$
\begin{aligned}
& \mathbf{C}_{1}=\int_{0}^{\pi / 2} \frac{2 \cos ^{2}(\varphi)+\left(1-\sigma(\varphi)^{2}\right) \sin ^{2}(\varphi)}{\sigma(\varphi)^{3}} \mathrm{~d} \varphi, \\
& \mathbf{C}_{2}=\int_{0}^{\pi / 2} \frac{2 \sin ^{2}(\varphi)+\left(1-\sigma(\varphi)^{2}\right) \cos ^{2}(\varphi)}{\sigma(\varphi)^{3}} \mathrm{~d} \varphi .
\end{aligned}
$$

Thus

$$
\Delta_{4}=\frac{2 k}{y_{3}^{2}}\left(\frac{3}{2}\left(\mathbf{A}_{1} \mathbf{C}_{2}-\mathbf{A}_{2} \mathbf{C}_{1}\right)+\frac{N C_{3}}{2 \pi k y_{3}^{2}}\left(\mathbf{A}_{1} \mathbf{B}_{2}-\mathbf{A}_{2} \mathbf{B}_{1}\right)+\frac{N}{2 \pi y_{3}}\left(\mathbf{C}_{1} \mathbf{B}_{2}-\mathbf{C}_{2} \mathbf{B}_{1}\right)\right) .
$$

Unfortunately, the three terms in the right-hand side are not all positive. Indeed, we recall that $\mathbf{B}_{i}=\mathbf{A}_{i}+\tilde{\mathbf{B}}_{i}$ for $i=1,2$, where $\tilde{\mathbf{B}}_{1}, \tilde{\mathbf{B}}_{2}$ are defined in (2.23). Similarly, $\mathbf{C}_{i}=\mathbf{A}_{i}-\tilde{\mathbf{C}}_{i}$, where

$$
\tilde{\mathbf{C}}_{1}=\int_{0}^{\pi / 2} \frac{\sin ^{2}(\varphi)}{\sigma(\varphi)} \mathrm{d} \varphi, \quad \tilde{\mathbf{C}}_{2}=\int_{0}^{\pi / 2} \frac{\cos ^{2}(\varphi)}{\sigma(\varphi)} \mathrm{d} \varphi .
$$


We also recall that $\mathbf{A}_{2}>\mathbf{A}_{1}, \mathbf{B}_{2}>\mathbf{B}_{1}, \mathbf{C}_{2}>\mathbf{C}_{1}$, but $\tilde{\mathbf{B}}_{1}>\tilde{\mathbf{B}}_{2}, \tilde{\mathbf{C}}_{1}>\tilde{\mathbf{C}}_{2}$. All these bounds follow from Lemma 2.5] as in (2.15). Thus

$$
\begin{aligned}
\mathbf{A}_{1} \mathbf{C}_{2}-\mathbf{A}_{2} \mathbf{C}_{1} & =\tilde{\mathbf{C}}_{1} \mathbf{A}_{2}-\tilde{\mathbf{C}}_{2} \mathbf{A}_{1}>0 \\
\mathbf{A}_{1} \mathbf{B}_{2}-\mathbf{A}_{2} \mathbf{B}_{1} & =\mathbf{A}_{1} \tilde{\mathbf{B}}_{2}-\mathbf{A}_{2} \tilde{\mathbf{B}}_{1}<0 \\
\mathbf{C}_{1} \mathbf{B}_{2}-\mathbf{C}_{2} \mathbf{B}_{1} & =\left(\mathbf{A}_{1} \mathbf{B}_{2}-\mathbf{A}_{2} \mathbf{B}_{1}\right)+\left(\tilde{\mathbf{C}}_{2} \mathbf{B}_{1}-\tilde{\mathbf{C}}_{1} \mathbf{B}_{2}\right)<0 .
\end{aligned}
$$

As $N \leq \pi y_{2}<\pi y_{3}$ and $C_{3}=(k N / \pi)<k y_{3}$, it follows from (3.31) that $\Delta_{4}>\left(2 k / y_{3}^{2}\right) \Delta_{5}$ where

$$
\Delta_{5}=\frac{3}{2}\left(\mathbf{A}_{1} \mathbf{C}_{2}-\mathbf{A}_{2} \mathbf{C}_{1}\right)+\frac{1}{2}\left(\mathbf{A}_{1} \mathbf{B}_{2}-\mathbf{A}_{2} \mathbf{B}_{1}\right)+\frac{1}{2}\left(\mathbf{C}_{1} \mathbf{B}_{2}-\mathbf{C}_{2} \mathbf{B}_{1}\right) .
$$

It remains to verify that $\Delta_{5}>0$. Since $\mathbf{B}_{i}=\mathbf{A}_{i}+\tilde{\mathbf{B}}_{i}$ and $\mathbf{C}_{i}=\mathbf{A}_{i}-\tilde{\mathbf{C}}_{i}$ for $i=1,2$, we have

$$
\begin{aligned}
\Delta_{5} & =\left(\tilde{\mathbf{C}}_{1} \mathbf{A}_{2}-\tilde{\mathbf{C}}_{2} \mathbf{A}_{1}\right)+\left(\mathbf{A}_{1} \tilde{\mathbf{B}}_{2}-\mathbf{A}_{2} \tilde{\mathbf{B}}_{1}\right)+\frac{1}{2}\left(\tilde{\mathbf{B}}_{1} \tilde{\mathbf{C}}_{2}-\tilde{\mathbf{B}}_{2} \tilde{\mathbf{C}}_{1}\right) \\
& =\left(\mathbf{A}_{2}-\mathbf{A}_{1}+\frac{\tilde{\mathbf{C}}_{1}-\tilde{\mathbf{C}}_{2}}{2}\right)\left(\tilde{\mathbf{C}}_{1}-\tilde{\mathbf{B}}_{1}\right)+\left(\mathbf{A}_{1}-\frac{\tilde{\mathbf{C}}_{1}}{2}\right)\left(\left(\tilde{\mathbf{C}}_{1}-\tilde{\mathbf{C}}_{2}\right)-\left(\tilde{\mathbf{B}}_{1}-\tilde{\mathbf{B}}_{2}\right)\right) .
\end{aligned}
$$

We claim that all terms in the right-hand side are now positive. Indeed, we already know that $\mathbf{A}_{2}-\mathbf{A}_{1}>0$ and $\tilde{\mathbf{C}}_{1}-\tilde{\mathbf{C}}_{2}>0$. Using (2.23) and (3.30) we obtain

$$
\tilde{\mathbf{C}}_{1}-\tilde{\mathbf{B}}_{1}=\int_{0}^{\pi / 2} \frac{(2+\sigma(\varphi)) \sin ^{2}(\varphi)-1}{(1+\sigma(\varphi))^{2}} \mathrm{~d} \varphi>\int_{0}^{\pi / 2} \frac{\sin ^{2}(\varphi)-\cos ^{2}(\varphi)}{(1+\sigma(\varphi))^{2}} \mathrm{~d} \varphi>0
$$

because $\varphi \mapsto(1+\sigma(\varphi))^{-2}$ is strictly increasing on $[0, \pi / 2]$. On the other hand, since $\sigma(\varphi) \leq 1$ we have

$$
\mathbf{A}_{1}>\int_{0}^{\pi / 2} \frac{1}{\sigma(\varphi)^{3}} \mathrm{~d} \varphi>\int_{0}^{\pi / 2} \frac{\sin ^{2}(\varphi)}{\sigma(\varphi)} \mathrm{d} \varphi=\tilde{\mathbf{C}}_{1},
$$

hence $\mathbf{A}_{1}-\tilde{\mathbf{C}}_{1} / 2>0$ a fortiori. Finally,

$$
\left(\tilde{\mathbf{C}}_{1}-\tilde{\mathbf{C}}_{2}\right)-\left(\tilde{\mathbf{B}}_{1}-\tilde{\mathbf{B}}_{2}\right)=\int_{0}^{\pi / 2} \frac{2+\sigma(\varphi)}{(1+\sigma(\varphi))^{2}}\left(\sin ^{2}(\varphi)-\cos ^{2}(\varphi)\right) \mathrm{d} \varphi>0,
$$

because $\varphi \mapsto(2+\sigma(\varphi)) /(1+\sigma(\varphi))^{2}$ is strictly increasing on $[0, \pi / 2]$. Thus $\Delta_{5}>0$, and the proof of Proposition 3.5 (hence of Proposition 3.1) is now complete.

\section{The focusing NLS case}

In this final section we show how the preceding results can be extended to the focusing NLS equation (1.1) with $\gamma=1$. In this case, Eq.(1.3) has quasi-periodic solutions for all values of $\omega$, but we restrict ourselves to the generic cases $\omega=1$ and $\omega=-1$, which we consider separately. 


\subsection{Counter-rotating waves $(\omega=1)$}

Proceeding as in Section 2, we first study the solutions of the stationary equation

$$
W_{x x}(x)+W(x)+|W(x)|^{2} W(x)=0, \quad x \in \mathbb{R} .
$$

This is again an integrable Hamiltonian system with conserved quantities

$$
J=\operatorname{Im}\left(\bar{W} W_{x}\right), \quad E=\frac{1}{2}\left|W_{x}\right|^{2}+\frac{1}{2}|W|^{2}+\frac{1}{4}|W|^{4} .
$$

In particular, the effective potential $V_{J}(r)=J^{2} /\left(2 r^{2}\right)+r^{2} / 2+r^{4} / 4$ is now strictly convex for any $J \in \mathbb{R}$. If we set $J=q\left(q^{2}-1\right)$ where $q \in \mathbb{R},|q| \geq 1$, then the unique minimum of $V_{J}$ is attained at $r=r_{q}=\sqrt{q^{2}-1}$ and has the value

$$
E_{-}(J)=V_{J}\left(\sqrt{q^{2}-1}\right)=\frac{1}{4}\left(q^{2}-1\right)\left(3 q^{2}+1\right) .
$$

It follows that (4.1) has quasi-periodic solutions if and only if $(J, E) \in D$, where

$$
D=\left\{(J, E) \in \mathbb{R}^{2} \mid E>E_{-}(J)\right\} .
$$

The period $T$ and the phase increment $\Phi$ of these solutions are given by

$$
T(J, E)=2 \sqrt{2} \int_{0}^{\pi / 2} \frac{\mathrm{d} \varphi}{\sqrt{s(\varphi)-y_{3}}}, \quad \Phi(J, E)=2 \sqrt{2} \int_{0}^{\pi / 2} \frac{J}{s(\varphi)} \frac{\mathrm{d} \varphi}{\sqrt{s(\varphi)-y_{3}}},
$$

where $s(\varphi)=y_{1} \cos ^{2}(\varphi)+y_{2} \sin ^{2}(\varphi)$ and $y_{3}<0 \leq y_{1}<y_{2}$ are the roots of the cubic polynomial $P(y)=-y^{3}-2 y^{2}+4 E y-2 J^{2}$. Similarly, the renormalized phase (2.9) satisfies

$$
\Psi(J, E)=-2 \sqrt{2} J \int_{0}^{\pi / 2} \frac{\mathrm{d} \varphi}{\sqrt{-y_{3}} \sqrt{s(\varphi)-y_{3}}\left(\sqrt{-y_{3}}+\sqrt{s(\varphi)-y_{3}}\right)} .
$$

In contrast with the defocusing case, the period $T(J, E)$ is now a decreasing function of the energy. The analogue of Proposition 2.4 is:

\section{Proposition 4.1}

i) $\frac{\partial T}{\partial E}(J, E)<0$ for all $(J, E) \in D$.

ii) $\frac{\partial \Psi}{\partial E}(J, E)=-\frac{\partial T}{\partial J}(J, E)>0$ for all $(J, E) \in D$ with $J>0$.

Proof. Differentiating $T$ with respect to $E$ and $J$ we obtain

$$
\frac{\partial T}{\partial E}=-A_{1} \frac{\partial y_{1}}{\partial E}-A_{2} \frac{\partial y_{2}}{\partial E}, \quad \frac{\partial T}{\partial J}=-A_{1} \frac{\partial y_{1}}{\partial J}-A_{2} \frac{\partial y_{2}}{\partial J}
$$

where

$$
A_{1}=\sqrt{2} \int_{0}^{\pi / 2} \frac{1+\cos ^{2}(\varphi)}{\left(s(\varphi)-y_{3}\right)^{3 / 2}} \mathrm{~d} \varphi, \quad A_{2}=\sqrt{2} \int_{0}^{\pi / 2} \frac{1+\sin ^{2}(\varphi)}{\left(s(\varphi)-y_{3}\right)^{3 / 2}} \mathrm{~d} \varphi
$$



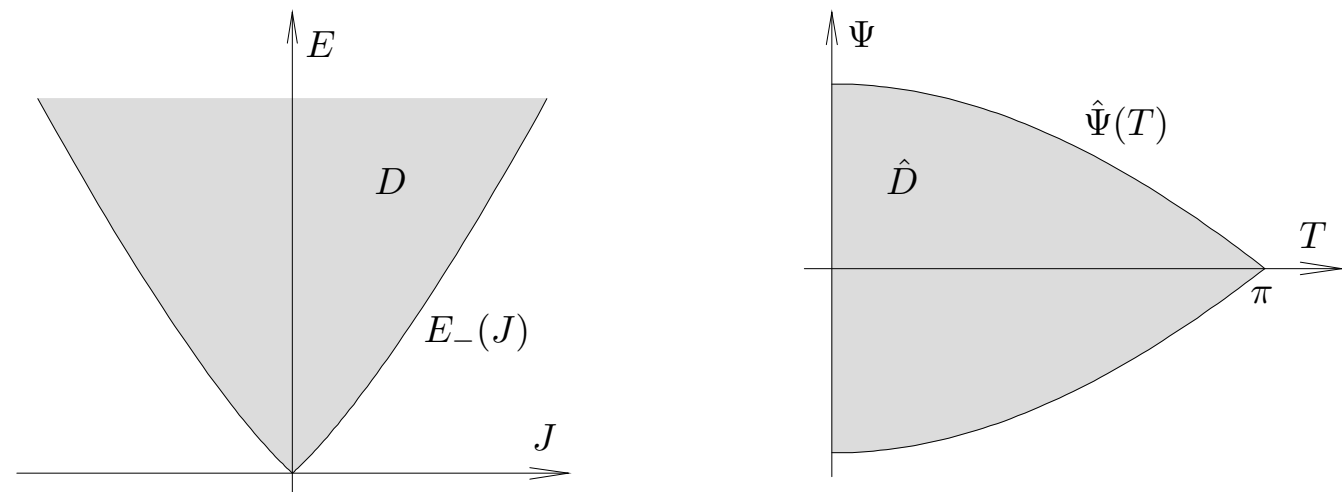

Fig. 5: Existence domain for the counter-rotating waves of the focusing NLS equation, in terms of the parameters $J, E$ (left) and $T, \Psi$ (right).

We remark that $\varphi \mapsto\left(s(\varphi)-y_{3}\right)^{-3 / 2}$ is strictly decreasing over $[0, \pi / 2]$. As

$$
A_{1}-A_{2}=\sqrt{2} \int_{0}^{\pi / 2} \frac{\cos ^{2}(\varphi)-\sin ^{2}(\varphi)}{\left(s(\varphi)-y_{3}\right)^{3 / 2}} \mathrm{~d} \varphi
$$

it follows from Lemma 2.5 that $A_{1}>A_{2}>0$. On the other hand, since

$$
\frac{\partial y_{i}}{\partial E}=-\frac{4 y_{i}}{-3 y_{i}^{2}-4 y_{i}+4 E}, \quad \frac{\partial y_{i}}{\partial J}=\frac{4 J}{-3 y_{i}^{2}-4 y_{i}+4 E}, \quad i=1,2,3,
$$

it is not difficult to verify that (2.12) still holds, whereas (2.13) is replaced by

$$
\frac{\partial y_{1}}{\partial J}>0, \quad \frac{\partial y_{2}}{\partial J}<0, \quad \frac{\partial y_{1}}{\partial J}+\frac{\partial y_{2}}{\partial J}=-\frac{\partial y_{3}}{\partial J}>0, \quad \text { if } J>0 .
$$

In particular, if $J>0$, we obtain

$$
-\frac{\partial T}{\partial J}=\left(A_{1}-A_{2}\right) \frac{\partial y_{1}}{\partial J}+A_{2}\left(\frac{\partial y_{1}}{\partial J}+\frac{\partial y_{2}}{\partial J}\right)>0
$$

which proves ii).

The main difficulty is the proof of $\mathrm{i}$ ), which requires a more sophisticated argument. As $y_{1}-y_{3}<$ $s(\varphi)-y_{3}<y_{2}-y_{3}$ for all $\varphi \in(0, \pi / 2)$, it is clear that

$$
\frac{c}{\left(y_{2}-y_{3}\right)^{3 / 2}}<A_{1}, A_{2}<\frac{c}{\left(y_{1}-y_{3}\right)^{3 / 2}}, \quad \text { where } \quad c=\frac{3 \pi \sqrt{2}}{4} \text {. }
$$

Using the upper bound for $A_{1}$ and the lower bound for $A_{2}$ we obtain from (4.5):

$$
-\frac{\partial T}{\partial E}>\frac{c}{\left(y_{1}-y_{3}\right)^{3 / 2}} \frac{\partial y_{1}}{\partial E}+\frac{c}{\left(y_{2}-y_{3}\right)^{3 / 2}} \frac{\partial y_{2}}{\partial E} .
$$

Replacing the derivatives $\partial y_{1} / \partial E$ and $\partial y_{2} / \partial E$ with their expressions (4.8), we see that the righthand side of (4.9) is positive if and only if

$$
P_{1} \stackrel{\text { def }}{=} y_{1}\left(y_{2}-y_{3}\right)^{3 / 2}\left(4 E-3 y_{2}^{2}-4 y_{2}\right)+y_{2}\left(y_{1}-y_{3}\right)^{3 / 2}\left(4 E-3 y_{1}^{2}-4 y_{1}\right)>0 .
$$


Now, since $4 E-3 y_{2}^{2}-4 y_{2}<0$ and $4 E-3 y_{1}^{2}-4 y_{1}>0$, this inequality is equivalent to

$$
\frac{y_{1}\left|4 E-3 y_{2}^{2}-4 y_{2}\right|}{y_{2}\left(4 E-3 y_{1}^{2}-4 y_{1}\right)}<\left(\frac{y_{1}-y_{3}}{y_{2}-y_{3}}\right)^{3 / 2}, \quad \text { where } 0<\frac{y_{1}-y_{3}}{y_{2}-y_{3}}<1 \text {. }
$$

Clearly, a stronger inequality is obtained if we replace the exponent $3 / 2$ by 2 in the right-hand side. Thus it is sufficient to show that $P_{2}>0$, where

$$
P_{2}=y_{1}\left(y_{2}-y_{3}\right)^{2}\left(4 E-3 y_{2}^{2}-4 y_{2}\right)+y_{2}\left(y_{1}-y_{3}\right)^{2}\left(4 E-3 y_{1}^{2}-4 y_{1}\right)>0 .
$$

To do that, we recall that $y_{1}, y_{2}, y_{3}$ are the roots of the cubic equation $y^{3}+2 y^{2}-4 E y+2 J^{2}=0$. In particular, we have $y_{1}+y_{2}+y_{3}=-2$ and $y_{1} y_{2} y_{3}=-2 J^{2}$. These relations allow to eliminate the variables $E$ and $y_{3}$ from the expression of $P_{2}$, which thus becomes a function of $y_{1}, y_{2}$ only. It is then convenient to set $y_{1}=y-z$ and $y_{2}=y+z$, where $0<z \leq y$. After a straightforward algebra, we obtain the final expression

$$
P_{2}=8 z^{2}\left((2+3 y)^{2}+z^{2}(3+4 y)\right)
$$

which shows that $P_{2}>0$. This concludes the proof.

Using Proposition 4.1 it is now easy to verify that the Hamiltonian system associated to (4.1) is non-degenerate, i.e. the determinant $\Delta(J, E)$ defined in (2.18) is always nonzero.

Proposition 4.2 For all $(J, E) \in D$ we have $\Delta(J, E)>0$.

Proof. Differentiating the renormalized phase $\Psi$ with respect to $J$ and $E$, we obtain as in (2.19)

$$
\frac{\partial \Psi}{\partial E}=B_{1} J \frac{\partial y_{1}}{\partial E}+B_{2} J \frac{\partial y_{2}}{\partial E}, \quad \frac{\partial \Psi}{\partial J}=B_{1} J \frac{\partial y_{1}}{\partial J}+B_{2} J \frac{\partial y_{2}}{\partial J}-B_{3}
$$

where

$$
\begin{gathered}
B_{1}=\sqrt{2} \int_{0}^{\pi / 2}\left\{\frac{1}{B_{2}} \frac{1 \sqrt{-y_{3}}+\sqrt{s(\varphi)-y_{3}}}{\left(-y_{3}\right)^{3 / 2}\left(s(\varphi)-y_{3}\right)^{1 / 2}} \frac{1}{\left(\sqrt{-y_{3}}+\sqrt{s(\varphi)-y_{3}}\right)^{2}}+\right. \\
\left.\frac{1}{\left(-y_{3}\right)^{1 / 2}\left(s(\varphi)-y_{3}\right)^{3 / 2}} \frac{\sqrt{-y_{3}}+2 \sqrt{s(\varphi)-y_{3}}}{\left(\sqrt{-y_{3}}+\sqrt{s(\varphi)-y_{3}}\right)^{2}}\left(1+\begin{array}{c}
\cos ^{2}(\varphi) \\
\sin ^{2}(\varphi)
\end{array}\right)\right\} \mathrm{d} \varphi, \\
B_{3}=2 \sqrt{2} \int_{0}^{\pi / 2} \frac{1}{\sqrt{-y_{3}} \sqrt{s(\varphi)-y_{3}}\left(\sqrt{-y_{3}}+\sqrt{s(\varphi)-y_{3}}\right)} \mathrm{d} \varphi=-\frac{\Psi}{J} .
\end{gathered}
$$

Thus replacing (4.5), (4.10) into (2.18) and proceeding as in the proof of Proposition 2.6] we obtain

$$
\Delta(J, E)=\left(A_{2} B_{1}-A_{1} B_{2}\right)\left(y_{2}-y_{1}\right) \frac{\partial y_{1}}{\partial J} \frac{\partial y_{2}}{\partial J}-B_{3} \frac{\partial T}{\partial E} .
$$

As $\partial T / \partial E<0$ by Proposition 4.1, it is thus sufficient to verify that $\Delta_{1}=A_{2} B_{1}-A_{1} B_{2}<0$. This inequality can be established using the same arguments as in the the defocusing case. Indeed, if we define (for $i=1,2) \mathbf{A}_{i}, \mathbf{B}_{i}$ by (2.22) and $\tilde{\mathbf{B}}_{i}$ by (2.23), we find $\Delta_{1}=2\left(-y_{3}\right)^{-4} \Delta_{2}$ where

$$
\Delta_{2}=\mathbf{A}_{2} \mathbf{B}_{1}-\mathbf{A}_{1} \mathbf{B}_{2}=\mathbf{A}_{2} \tilde{\mathbf{B}}_{1}-\mathbf{A}_{1} \tilde{\mathbf{B}}_{2}
$$


Now, we observe that $\varphi \mapsto \sigma(\varphi)=\left(1-y_{3}^{-1} s(\varphi)\right)^{1 / 2}$ is increasing over $[0, \pi / 2]$, because $y_{3}<0$. Using Lemma 2.5] we deduce that $\mathbf{A}_{1}>\mathbf{A}_{2}>0$ and $\tilde{\mathbf{B}}_{2}>\tilde{\mathbf{B}}_{1}>0$, hence $\Delta_{2}<0$.

As in Section 2. Propositions 4.14 .2 allow to determine the range of values of the period $T$ and the renormalized phase $\Psi$. We find that $(T, \Psi): D \rightarrow \hat{D}$ is a smooth diffeomorphism, where

$$
\hat{D}=\left\{(T, \Psi) \in \mathbb{R}^{2}|0<T<\pi,| \Psi \mid<\hat{\Psi}(T)\right\}, \quad \text { where } \quad \hat{\Psi}(T)=\pi-\left(\frac{T^{2}+2 \pi^{2}}{3}\right)^{1 / 2} .
$$

The domains $D$ and $\hat{D}$ are represented in Fig. 5 .

Now, we fix $(J, E) \in D$ and we study the stability of the periodic wave $U_{J, E}(x, t)=\mathrm{e}^{-\mathrm{i} t} W_{J, E}(x)$, where $W_{J, E}$ is a solution of (4.1) satisfying (4.2). As in Section 3 we set $W_{J, E}(x)=\mathrm{e}^{\mathrm{i} p x} Q_{J, E}(2 k x)$, where $k, \ell$ are defined in (2.27) and $p=k+\ell$. The discussion follows exactly the same lines as in the defocusing case, so we shall just mention the main differences. The function $Q(z, t)$ defined in (3.1) satisfies the evolution equation

$$
\mathrm{i} Q_{t}+4 \mathrm{i} p k Q_{z}+4 k^{2} Q_{z z}+\left(1-p^{2}\right) Q+|Q|^{2} Q=0,
$$

and the corresponding energy functional reads

$$
\mathcal{E}(Q)=\int_{0}^{2 \pi}\left(2 k^{2}\left|Q_{z}(z)\right|^{2}-\frac{1}{4}|Q(z)|^{4}\right) \mathrm{d} z .
$$

In particular, if we define $\mathcal{E}_{J, E}$ by (3.6), the second variation becomes

$$
H_{J, E}=\mathcal{E}_{J, E}^{\prime \prime}\left(Q_{J, E}\right)=-4 k^{2} \partial_{z z}-4 \mathrm{i} p k \partial_{z}-\left(1-p^{2}\right)-\left|Q_{J, E}\right|^{2}-2 Q_{J, E} \otimes Q_{J, E} .
$$

As in the defocusing case, we rely on the result found for small waves in [12, Remark A.2] and conclude that, when $J=0$ and $E>0$ is sufficiently small, the operator $H_{J, E}$ acting on $X_{0}$ has exactly three eigenvalues $\left\{\lambda_{1}, \lambda_{2}, 0\right\}$ in a neighborhood of the origin, where

$$
\lambda_{1}(E)=-3 E+\mathcal{O}\left(E^{2}\right), \quad \lambda_{2}(E)=E+\mathcal{O}\left(E^{2}\right), \quad \text { as } E \rightarrow 0 .
$$

The other eigenvalues of $H_{J, E}$ are positive and bounded away from zero. On the other hand, we know that zero is an eigenvalue of $H_{J, E}$ of multiplicity exactly two for all $(J, E) \in D$. Indeed, this property was established in Section 3 by a general argument which uses only the symmetries of (2.1) and not the particular form of the nonlinearity. By continuity, it follows that $H_{J, E}$ has exactly one negative eigenvalue for all $(J, E) \in D$, so that Proposition 3.2 remains valid in the present case.

Finally, we recall that the equilibrium $Q_{J, E}$ of (4.11) is a member of a two-parameter family of travelling and rotating waves of the form (3.11). For sufficiently small $(\omega, c)$, the wave profile is given by $Q_{J, E}^{\omega, c}(z)=\lambda Q_{J^{\prime}, E^{\prime}}(z)$, where $\lambda$ is defined by (3.12) and $\left(J^{\prime}, E^{\prime}\right) \in D$ is the only point in a neighborhood of $(J, E)$ such that (3.13) holds. Let $\mathcal{H}_{J, E}$ be the Hessian matrix of the function $d_{J, E}$ defined by (3.18), (3.16). In view of Proposition 3.2 the general results of [16] imply:

Proposition 4.3 For all $(J, E) \in D$ such that $\operatorname{det}\left(\mathcal{H}_{J, E}\right)<0$, the periodic wave $Q_{J, E}$ is a stable equilibrium of (4.11) in the sense of Proposition 3.1. 


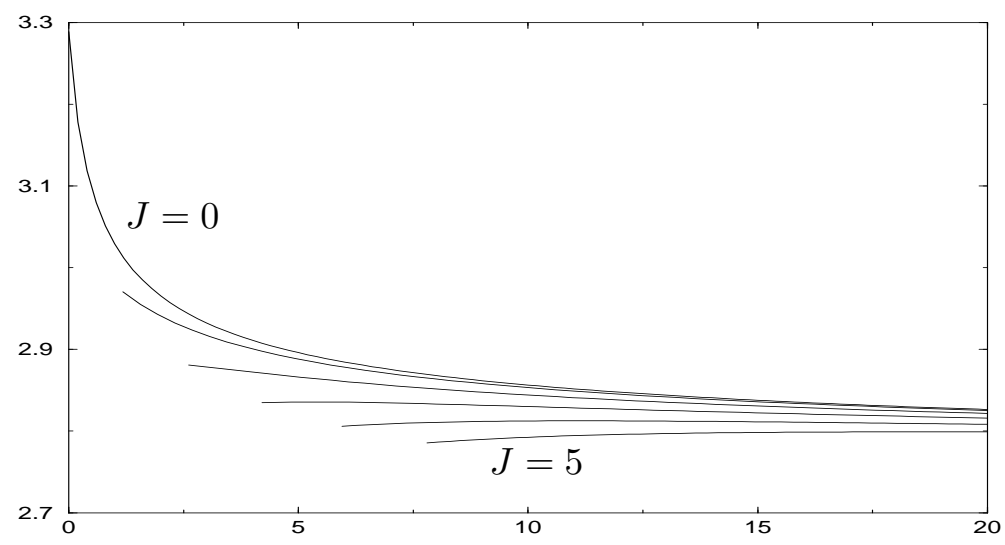

Fig. 6: The quantity $-\operatorname{det}\left(\mathcal{H}_{J, E}\right)$ is represented as a function of $E \in\left[E_{-}(J), 20\right]$ for $J=0,1, \ldots, 5$. Similar curves are obtained for higher values of $J$, thus indicating that $\operatorname{det}\left(\mathcal{H}_{J, E}\right)$ is always negative.

Proposition 4.3 is a conditional stability result, since it applies under the assumption that $\operatorname{det}\left(\mathcal{H}_{J, E}\right)<0$. This condition is satisfied at least for $(J, E)$ sufficiently close to zero, because we know from 12 that

$$
\mathcal{H}_{J, E}=\frac{\pi}{3}\left(\begin{array}{cc}
2 & 1 \\
1 & -1
\end{array}\right)(\mathbf{1}+\mathcal{O}(E)), \quad \text { as } \quad(J, E) \rightarrow(0,0) \text { in } D .
$$

Moreover, the Hessian matrix $\mathcal{H}_{J, E}$ is easy to evaluate numerically for any $(J, E) \in D$, and its determinant appears to be always negative (see Fig. 6). Thus we conjecture that $\operatorname{det}\left(\mathcal{H}_{J, E}\right)<0$ for all $(J, E) \in D$. This property can probably be established rigorously using similar arguments as in the proof of Proposition 3.5. but the modifications are not straightforward.

\subsection{Corotating waves $(\omega=-1)$}

Finally we study the corotating waves of the focusing nonlinear Schrödinger equation. Our starting point is the stationary equation

$$
W_{x x}(x)-W(x)+|W(x)|^{2} W(x)=0, \quad x \in \mathbb{R} .
$$

The invariants of this Hamiltonian system have the following expressions:

$$
J=\operatorname{Im}\left(\bar{W} W_{x}\right), \quad E=\frac{1}{2}\left|W_{x}\right|^{2}-\frac{1}{2}|W|^{2}+\frac{1}{4}|W|^{4} .
$$

It is convenient to use the parametrization $J=q\left(1+q^{2}\right)$, where $q \in \mathbb{R}$. If $J \neq 0$, the effective potential $V_{J}(r)=J^{2} /\left(2 r^{2}\right)-r^{2} / 2+r^{4} / 4$ has a unique critical point at $r=r_{q}=\sqrt{1+q^{2}}$, where $V_{J}$ attains its global minimum:

$$
E_{-}(J)=V_{J}\left(\sqrt{1+q^{2}}\right)=\frac{1}{4}\left(q^{2}+1\right)\left(3 q^{2}-1\right) .
$$

In that case, Eq.(4.13) has quasi-periodic solutions for all $E>E_{-}(J)$. If $J=0$, the double-well potential $V_{0}(r)$ has two minima at $r= \pm 1$ and a local maximum at $r=0$. It follows that (4.13) 
has (real) periodic solutions if $-1 / 4<E<0$ (dnoidal waves) and if $E>0$ (cnoidal waves). Summarizing, the parameter domain where quasi-periodic solutions of (4.13) exist is

$$
D=\left\{(J, E) \in \mathbb{R}^{2} \mid E>E_{-}(J)\right\} \backslash\{(0,0)\} .
$$

In the exceptional case $(J, E)=(0,0)$, Eq. (4.13) has the pulse-like solution $W(x)=\sqrt{2} / \cosh (x)$ which corresponds to the solitary wave of the focusing NLS equation.

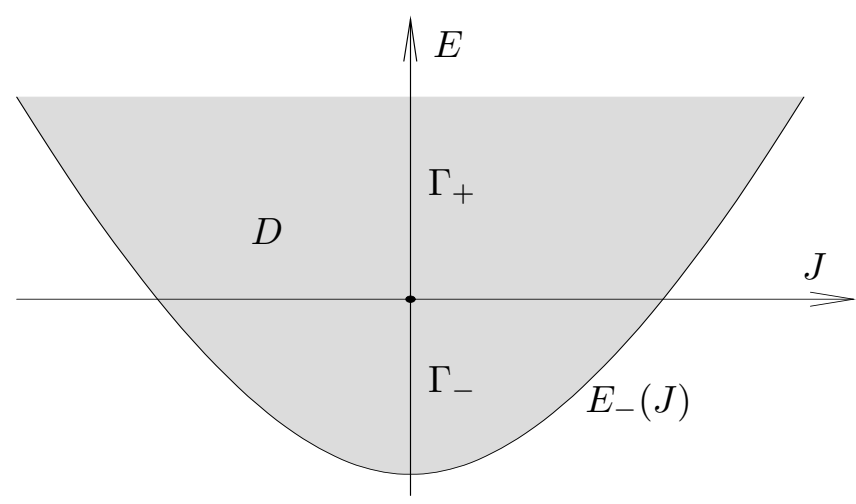

Fig. 7: Existence domain for the corotating waves of the focusing NLS equation. The origin $(J, E)=(0,0)$ corresponds to the solitary wave. The half-line $\Gamma_{+}$(cnoidal waves) and the segment $\Gamma_{-}$(dnoidal waves) are the discontinuity lines of the phases $\Phi$ and $\Psi$, respectively.

If $(J, E) \in D$, the period $T$, the phase increment $\Phi$, and the renormalized phase $\Psi$ of the quasiperiodic solutions are given by the formulas (4.3), (4.4), where $s(\varphi)=y_{1} \cos ^{2}(\varphi)+y_{2} \sin ^{2}(\varphi)$ and $y_{3} \leq 0 \leq y_{1}<y_{2}$ are the roots of the cubic polynomial $P(y)=-y^{3}+2 y^{2}+4 E y-2 J^{2}$. It is important to realize that the phase $\Phi$ is continuous on $D$, except on the half-line

$$
\Gamma_{+}=\left\{(0, E) \in \mathbb{R}^{2} \mid E>0\right\}=\left\{(J, E) \in D \mid y_{1}=0\right\},
$$

(see Fig. 7). Similarly, the renormalized phase $\Psi$ is continuous on $D$ except for a $2 \pi$-jump on the line segment

$$
\Gamma_{-}=\left\{(0, E) \in \mathbb{R}^{2} \mid-1 / 4<E<0\right\}=\left\{(J, E) \in D \mid y_{3}=0\right\} .
$$

We shall thus use either $\Phi$ or $\Psi$ depending on the parameter region under consideration.

Unlike in the previous cases, the period $T$ is no longer a monotone function of the energy $E$. This is intuitively clear, as we expect that $T \rightarrow+\infty$ as $(J, E) \rightarrow(0,0)$. In fact, one can prove:

Lemma 4.4 The period $T(J, E)$ satisfies

$$
\frac{\partial T}{\partial E}(0, E)>0 \text { for }-1 / 4<E<0, \quad \text { and } \quad \frac{\partial T}{\partial E}(0, E)<0 \text { for } E>0 .
$$

Proof. As in (4.5), we have

$$
\frac{\partial T}{\partial E}=-A_{1} \frac{\partial y_{1}}{\partial E}-A_{2} \frac{\partial y_{2}}{\partial E}
$$


where the coefficients $A_{1}, A_{2}$ are defined in (4.6). The only difference with the previous case is that the quantities $y_{1}, y_{2}, y_{3}$ which appear in (4.6) are the roots of a different polynomial. If $J=0$ and $E>0$, then $y_{1}=0, y_{2}=1+\sqrt{1+4 E}, y_{3}=1-\sqrt{1+4 E}$, hence

$$
\frac{\partial T}{\partial E}(0, E)=-A_{2} \frac{2}{\sqrt{1+4 E}}<0 .
$$

If $J=0$ and $-1 / 4<E<0$, then $y_{1}=1-\sqrt{1+4 E}, y_{2}=1+\sqrt{1+4 E}, y_{3}=0$, so that

$$
\frac{\partial T}{\partial E}(0, E)=\frac{2}{\sqrt{1+4 E}}\left(A_{1}-A_{2}\right)>0,
$$

because $A_{1}>A_{2}$ by (4.7).

On the other hand, the period $T$ is still a monotone function of $J$ if $J>0$. As in Proposition 4.1 one can prove that

$$
\frac{\partial \Psi}{\partial E}(J, E)=\frac{\partial \Phi}{\partial E}(J, E)=-\frac{\partial T}{\partial J}(J, E)>0, \quad \text { if } J>0 .
$$

The important quantity is again

$$
\Delta(J, E)=\operatorname{det}\left(\begin{array}{ll}
\frac{\partial T}{\partial E} & \frac{\partial \Phi}{\partial E} \\
\frac{\partial T}{\partial J} & \frac{\partial \Phi}{\partial J}
\end{array}\right)(J, E)=\operatorname{det}\left(\begin{array}{ll}
\frac{\partial T}{\partial E} & \frac{\partial \Psi}{\partial E} \\
\frac{\partial T}{\partial J} & \frac{\partial \Psi}{\partial J}
\end{array}\right)(J, E) .
$$

Here the first determinant in the right-hand side is meaningful if $(J, E) \notin \Gamma_{+}$, and the second one if $(J, E) \notin \Gamma_{-}$.

Proposition 4.5 For all $(J, E) \in D$ we have $\Delta(J, E)>0$.

Proof. Fix $(J, E) \in D$. If $(\partial T / \partial E)(J, E)<0$, we know from Lemma 4.4 that $(J, E) \notin \Gamma_{-}$. Thus, using the second determinant in (4.15) and proceeding exactly as in Proposition 4.2, we obtain $\Delta(J, E)>0$.

We now assume that $(\partial T / \partial E)(J, E) \geq 0$, so that $(J, E) \notin \Gamma_{+}$by Lemma 4.4. Differentiating the expression of $\Phi$ in (4.3) with respect to $E$ and $J$, we obtain

$$
\frac{\partial \Phi}{\partial E}=-\mathcal{B}_{1} J \frac{\partial y_{1}}{\partial E}-\mathcal{B}_{2} J \frac{\partial y_{2}}{\partial E}, \quad \frac{\partial \Phi}{\partial J}=-\mathcal{B}_{1} J \frac{\partial y_{1}}{\partial J}-\mathcal{B}_{2} J \frac{\partial y_{2}}{\partial J}+\mathcal{B}_{3},
$$

where

$$
\begin{aligned}
\mathcal{B}_{1} & =\sqrt{2} \int_{0}^{\pi / 2}\left(\frac{2 \cos ^{2}(\varphi)}{s(\varphi)^{2} \sqrt{s(\varphi)-y_{3}}}+\frac{1+\cos ^{2}(\varphi)}{s(\varphi)\left(s(\varphi)-y_{3}\right)^{3 / 2}}\right) \mathrm{d} \varphi=\mathcal{B}_{11}+\mathcal{B}_{12}, \\
\mathcal{B}_{2} & =\sqrt{2} \int_{0}^{\pi / 2}\left(\frac{2 \sin ^{2}(\varphi)}{s(\varphi)^{2} \sqrt{s(\varphi)-y_{3}}}+\frac{1+\sin ^{2}(\varphi)}{s(\varphi)\left(s(\varphi)-y_{3}\right)^{3 / 2}}\right) \mathrm{d} \varphi=\mathcal{B}_{21}+\mathcal{B}_{22}, \\
\mathcal{B}_{3} & =2 \sqrt{2} \int_{0}^{\pi / 2} \frac{1}{s(\varphi) \sqrt{s(\varphi)-y_{3}}} \mathrm{~d} \varphi=\frac{\Phi}{J} .
\end{aligned}
$$

Then, using (4.5), (4.16), and the first determinant in (4.15), we find as in Proposition [2.6

$$
\Delta(J, E)=\left(A_{1} \mathcal{B}_{2}-A_{2} \mathcal{B}_{1}\right)\left(y_{2}-y_{1}\right) \frac{\partial y_{1}}{\partial J} \frac{\partial y_{2}}{\partial J}+\mathcal{B}_{3} \frac{\partial T}{\partial E} .
$$


As $(\partial T / \partial E) \geq 0$ by assumption, it is sufficient to verify that $\Delta_{1} \stackrel{\text { def }}{=} A_{2} \mathcal{B}_{1}-A_{1} \mathcal{B}_{2}>0$.

To do that, we observe that $\Delta_{1}=\Delta_{11}+\Delta_{12}$ where

$$
\Delta_{11}=A_{2} \mathcal{B}_{11}-A_{1} \mathcal{B}_{21}, \quad \Delta_{12}=A_{2} \mathcal{B}_{12}-A_{1} \mathcal{B}_{22},
$$

and we prove separately that $\Delta_{11}>0$ and $\Delta_{12}>0$. Both inequalities are easy consequences of Lemma 2.5. Indeed, $\Delta_{11}>0$ is equivalent to $\left(A_{1}-A_{2}\right) /\left(A_{1}+A_{2}\right)<\left(\mathcal{B}_{11}-\mathcal{B}_{21}\right) /\left(\mathcal{B}_{11}+\mathcal{B}_{21}\right)$, or explicitly

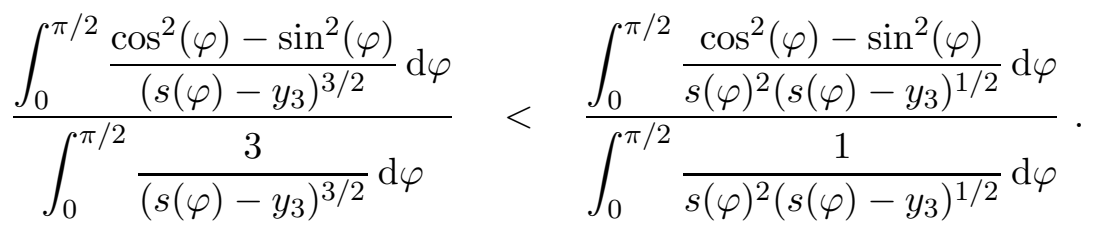

A stronger inequality (without the factor 3 in the denominator of the left-hand side) is obtained from Lemma 2.5 by choosing $I=[0, \pi / 2], f(\varphi)=\cos ^{2}(\varphi)-\sin ^{2}(\varphi), g(\varphi)=\left(s(\varphi)-y_{3}\right) /(s(\varphi))^{2}$ and

$$
\mathrm{d} \mu=\frac{1}{\mathcal{N}} \frac{\mathrm{d} \varphi}{\left(s(\varphi)-y_{3}\right)^{3 / 2}}, \quad \text { where } \quad \mathcal{N}=\int_{0}^{\pi / 2} \frac{\mathrm{d} \varphi}{\left(s(\varphi)-y_{3}\right)^{3 / 2}} .
$$

Indeed $\int_{I} f g \mathrm{~d} \mu>\left(\int_{I} f \mathrm{~d} \mu\right)\left(\int_{I} g \mathrm{~d} \mu\right)$ because $f, g$ are strictly decreasing over $I$. Thus $\Delta_{11}>0$, and the same argument with $g(\varphi)=1 / s(\varphi)$ shows that $\Delta_{12}>0$.

To conclude this section, we fix $(J, E) \in D$ and we study the stability of the periodic wave $U_{J, E}(x, t)=\mathrm{e}^{\mathrm{i} t} W_{J, E}(x)$, where $W_{J, E}=\mathrm{e}^{\mathrm{i} p x} Q_{J, E}(2 k x)$ is a solution of (4.13) satisfying (4.14). Setting $U(x, t)=\mathrm{e}^{\mathrm{i}(p x+t)} Q(2 k x, t)$, we obtain from (1.1) with $\gamma=1$ the evolution equation

$$
\mathrm{i} Q_{t}+4 \mathrm{i} p k Q_{z}+4 k^{2} Q_{z z}-\left(1+p^{2}\right) Q+|Q|^{2} Q=0 .
$$

The wave profile $Q_{J, E}$ is a critical point of the modified energy

$$
\mathcal{E}_{J, E}(Q)=\mathcal{E}(Q)+\left(1+p^{2}\right) N(Q)-4 p k M(Q),
$$

where $\mathcal{E}$ is defined in (4.12) and $N, M$ in (3.4). The second variation of $\mathcal{E}_{J, E}$ at $Q_{J, E}$ is

$$
H_{J, E}=\mathcal{E}_{J, E}^{\prime \prime}\left(Q_{J, E}\right)=-4 k^{2} \partial_{z z}-4 \mathrm{i} p k \partial_{z}+\left(1+p^{2}\right)-\left|Q_{J, E}\right|^{2}-2 Q_{J, E} \otimes Q_{J, E} .
$$

Again, one can prove that Proposition 3.2 still holds in the present case. The fact that zero is always a double eigenvalue of $H_{J, E}$ is established as in Section 3 and a direct calculation for small amplitude periodic waves (in a neighborhood of a plane wave) shows that $H_{J, E}$ has exactly one negative eigenvalue.

As in the previous cases, the equilibrium $Q_{J, E}$ of (4.11) is a member of a two-parameter family of travelling and rotating waves of the form (3.11). For sufficiently small $(\omega, c)$, the wave profile is given by $Q_{J, E}^{\omega, c}(z)=\lambda Q_{J^{\prime}, E^{\prime}}(z)$, where $\lambda$ is defined by (3.12) and $\left(J^{\prime}, E^{\prime}\right) \in D$ is the only point in a neighborhood of $(J, E)$ such that

$$
\omega=\left(1+p^{2}\right)-\lambda^{2}\left(1+p^{2}\right), \quad c=4 \lambda^{2} k^{\prime} p^{\prime}-4 k p .
$$

If $\mathcal{H}_{J, E}$ denotes the Hessian matrix of the function $d_{J, E}$ defined by (3.18), (3.16), the results of [16] imply: 
Proposition 4.6 For all $(J, E) \in D$ such that $\operatorname{det}\left(\mathcal{H}_{J, E}\right)<0$, the periodic wave $Q_{J, E}$ is a stable equilibrium of (4.17) in the sense of Proposition 3.1.

As in Section 4.1, we conjecture that $\operatorname{det}\left(\mathcal{H}_{J, E}\right)<0$ for all $(J, E) \in D$. This inequality is true at least for small amplitude periodic waves (in a neighborhood of a plane wave), and numerical calculations indicate that it remains valid over the whole parameter domain $D$. In the particular case where $(J, E) \in \Gamma_{-}$(dnoidal waves), the orbital stability with respect to periodic perturbations has been established in [1]. Remark that Proposition 4.6] does apply in the case where $(J, E) \in \Gamma_{+}$ (cnoidal waves), which is not covered by the results of [1], but as is explained in the introduction this is because we use in fact a more restricted class of perturbations.

\section{References}

[1] J. Angulo Pava. Nonlinear stability of periodic travelling wave solutions to the Schrödinger and the modified Korteweg-de Vries equations. J. Diff. Equations, to appear.

[2] J. Angulo Pava, J. L. Bona, and M. Scialom. Stability of cnoidal waves. Preprint (2006).

[3] T. Benjamin. The stability of solitary waves. Proc. Roy. Soc. London Ser. A 328 (1972), $153-183$.

[4] J. Bona. On the stability theory of solitary waves. Proc. Roy. Soc. London Ser. A 344 (1975), $363-374$.

[5] Th. Bridges and G. Rowlands. Instability of spatially quasi-periodic states of the GinzburgLandau equation. Proc. Roy. Soc. London Ser. A 444 (1994), 347-362.

[6] J. Bronski and Z. Rapti. Modulational instability for Nonlinear Schrödinger equations with a periodic potential. Dynamics of PDE 2 (2005), 335-355.

[7] Th. Cazenave and P.-L. Lions. Orbital stability of standing waves for some nonlinear Schrödinger equations. Comm. Math. Phys. 85 (1982), 549-561.

[8] Th. Cazenave and F. Weissler. The Cauchy problem for the nonlinear Schrödinger equation in $H^{1}$. Manuscripta Math. 61 (1988), 477-494.

[9] A. Doelman, R.A. Gardner, and C.K.R.T. Jones. Instability of quasiperiodic solutions of the Ginzburg-Landau equation. Proc. Roy. Soc. Edinburgh Sect. A 125 (1995), 501-517.

[10] J.-P. Eckmann, Th. Gallay, and C.E. Wayne. Phase slips and the Eckhaus instability. Nonlinearity 8 (1995), 943-961.

[11] Th. Gallay. Existence et stabilité des fronts dans l'équation de Ginzburg-Landau à une dimension. PhD Thesis, Université de Genève, 1994.

[12] Th. Gallay and M. Hărăguş. Stability of small periodic waves for the nonlinear Schrödinger equations. Preprint (2006). 
[13] J. Ginibre and G. Velo. On a class of nonlinear Schrödinger equations. I. The Cauchy problem, general case. J. Funct. Anal. 32 (1979), 1-32.

[14] J. Ginibre and G. Velo. The global Cauchy problem for the nonlinear Schrödinger equation revisited. Ann. Inst. H. Poincaré Anal. Non Linéaire 2 (1985), 309-327.

[15] M. Grillakis, J. Shatah, and W. Strauss. Stability theory of solitary waves in the presence of symmetry. I. J. Funct. Anal. 74 (1987), 160-197.

[16] M. Grillakis, J. Shatah, and W. Strauss. Stability theory of solitary waves in the presence of symmetry. II. J. Funct. Anal. 94 (1990), 308-348.

[17] T. Kato. On nonlinear Schrödinger equations. Ann. Inst. H. Poincaré Phys. Théor. 46 (1987), $113-129$.

[18] M. Oh, B. Sandstede, and A. Yew. Stability of cnoidal waves for the focussing nonlinear Schrödinger equation with potential. In preparation.

[19] G. Rowlands. On the stability of solutions of the Non-linear Schrödinger equation. IMA $J$ Appl Math. 13 (1974), 367-377.

[20] C. Sulem and P.-L. Sulem. The nonlinear Schrödinger equation. Self-focusing and wave collapse. Applied Mathematical Sciences 139, Springer, New York, 1999.

[21] M. Weinstein. Lyapunov stability of ground states of nonlinear dispersive evolution equations. Comm. Pure Appl. Math. 39 (1986), 51-67.

[22] P. Zhidkov. Korteweg-de Vries and nonlinear Schrödinger equations: qualitative theory. Lecture Notes in Mathematics 1756, Springer-Verlag, Berlin, 2001. 